\title{
Temporal dynamics of sequential motor activation in a dual-prime paradigm: Insights from conditional accuracy and hazard functions
}

\author{
Maximilian P. Wolkersdorfer ${ }^{1} \cdot$ Sven Panis $^{1} \cdot$ Thomas Schmidt $^{1}$ \\ Published online: 12 March 2020 \\ (C) The Author(s) 2020
}

\begin{abstract}
In response priming experiments, a participant has to respond as quickly and as accurately as possible to a target stimulus preceded by a prime. The prime and the target can either be mapped to the same response (consistent trial) or to different responses (inconsistent trial). Here, we investigate the effects of two sequential primes (each one either consistent or inconsistent) followed by one target in a response priming experiment. We employ discrete-time hazard functions of response occurrence and conditional accuracy functions to explore the temporal dynamics of sequential motor activation. In two experiments (small- $N$ design, 12 participants, 100 trials per cell and subject), we find that (1) the earliest responses are controlled exclusively by the first prime if primes are presented in quick succession, (2) intermediate responses reflect competition between primes, with the second prime increasingly dominating the response as its time of onset is moved forward, and (3) only the slowest responses are clearly controlled by the target. The current study provides evidence that sequential primes meet strict criteria for sequential response activation. Moreover, it suggests that primes can influence responses out of a memory buffer when they are presented so early that participants are forced to delay their responses.
\end{abstract}

Keywords Feedforward sweep $\cdot$ Response priming $\cdot$ Event history analysis $\cdot$ Reaction- time analysis $\cdot$ Visuomotor

Priming paradigms are very popular in many fields of cognitive psychology to study how exposure to a prime stimulus influences the response to a subsequently presented target stimulus. In general, the representations that mediate priming can be located at perceptual (Wiggs \& Martin, 1998), conceptual/semantic (e.g., Schacter \& Buckner, 1998), lexical (e.g., Fernández-López, Marcet, \& Perea, 2019), phonological (e.g., Ferrand \& Grainger, 1992), and/or motor response levels (e.g., Rosenbaum, 1983). In this paper we focus on the socalled response priming paradigm (Klotz \& Neumann, 1999; Klotz \& Wolff, 1995; Vorberg, Mattler, Heinecke, Schmidt, \&

Maximilian P. Wolkersdorfer

max.wolkersdorfer@sowi.uni-kl.de

Sven Panis

sven.panis@sowi.uni-kl.de

Thomas Schmidt

thomas.schmidt@sowi.uni-kl.de

1 Faculty of Social Sciences, Experimental Psychology Unit, University of Kaiserslautern, Erwin-Schrödinger-Str. Geb. 57, D-67663 Kaiserslautern, Germany
Schwarzbach, 2003). In a typical response priming experiment, a participant has to respond as quickly and as accurately as possible to a target stimulus preceded by a (masked or unmasked) prime stimulus. The prime and the target can either be mapped to the same response (consistent trial) or to different responses (inconsistent trial). While consistent trials typically show accelerated and more accurate responses, inconsistent trials show decelerated and less accurate responses, respectively. The differences between consistent and inconsistent trials in both mean reaction times (RTs) and overall error rates (ERs) define the response priming effect. Characteristically, this priming effect increases linearly with stimulus-onset asynchrony (SOA) for SOAs of up to about $100 \mathrm{~ms}$ (Vorberg et al., 2003). Response priming effects are believed to be mostly mediated by motor response conflicts (Schmidt, Haberkamp, \& Schmidt, 2011; Schmidt, 2002). However, how a rapid sequence of visual stimuli is processed and converted into motor action is still under debate. In order to gain insights into the covert temporal dynamics of our visual system and the online transfer of visual signals into overt behavior, we employ event history analysis, a longitudinal technique to perform a distributional analysis. 


\section{Multiple-prime paradigm}

What if instead of only one prime, a sequence of primes is preceding a target stimulus? A number of previous studies have touched upon this question. Jaśkowski, Skalska, and Verleger (2003) presented five pairs of squares sequentially, with an SOA of $35 \mathrm{~ms}$, so that each stimulus masked the previous one via metacontrast. The last and largest pair was the target, and observers had to decide whether the left or right square contained a gap. The first four pairs could serve as masked primes that contained a gap in the same (consistent) or opposite (inconsistent) square as the target. They found that the priming effect in mean correct RT increases with the number of primes presented in a sequence of successively masked stimuli. Because all of the primes within a single trial were either consistent or inconsistent to the target, this result would be expected from the accumulation of prime information (Miller, 1982). Jaśkowski et al. (2003) concluded that "motor activation evoked by a series of primes does accumulate, facilitating or inhibiting motor responses to the target" (p. 913).

Similarly, Breitmeyer and Hanif (2008) showed that when two successively presented prime stimuli are both consistent to a target in terms of shape (square versus diamond), mean RTs are faster than when only one of the two primes is consistent. Furthermore, they found that the priming effects from the second prime dominate over those of the first prime. That is, if the first prime was consistent and the second inconsistent to the target (condition "CI"), mean RT increased much more than when the first prime was inconsistent and the second consistent (condition "IC"). This contradicts the idea that due to the longer Prime1-target SOA, the first prime should cause a larger priming effect than the succeeding second prime. They argue that the second prime instead updates and overrides the effects of the first prime.

Grainger, Scharnowski, Schmidt, and Herzog (2013) employed two 20-ms Vernier stimuli as primes. In a series of experiments, they found that (1) two primes presented in immediate succession at the same location integrate before activating a motor response, and do not cause sequential activation; (2) two identical primes yield larger priming effects than single primes; (3) one consistent and one inconsistent prime presented simultaneously at different locations cancel each other's effects. More importantly, in the varying-primes condition of their Experiment 3, they presented two lateralized Vernier primes and a central Vernier target, kept the Prime 1-target SOA constant at $200 \mathrm{~ms}$, and varied the interprime interval (and thus also Prime 2-target ISI). For interprime intervals of 30 and $80 \mathrm{~ms}$ Prime 2 clearly dominated, but for an interprime interval of $150 \mathrm{~ms}$ (and a corresponding Prime $2-\operatorname{target}$ ISI of $30 \mathrm{~ms}$ ) Prime 1 dominated slightly. The authors propose that all visual stimuli enter a time-selective buffer stage, integrate, and only then initiate a motor response. Instead of activating their associated responses in strict sequence, their joint impact is determined by their relative dominance in the motor buffer.

However, it has been suggested that -in the context of response-conflict paradigms such as response priming and flanker effects-sequential visual stimuli elicit sequential feedforward sweeps (Bullier, 2001; Lamme \& Roelfsema, 2000; VanRullen \& Koch, 2003). These fast and bottom-up processes can activate motor responses in a strictly sequential manner (T. Schmidt et al., 2011). Moreover, since both prime and target in a response priming paradigm activate their respective motor responses, response conflict arises if prime and target are inconsistent, thus leading to an increase in RT (Schmidt, 2014). Several studies have demonstrated the existence of this feedforward and sequential activation, in both neuronal activity, such as lateralized readiness potentials (Eimer \& Schlaghecken, 1998; Vath \& Schmidt, 2007), and overt behavior, such as the time course of pointing movements (Schmidt \& Schmidt, 2010; Schmidt, 2002; Schmidt \& Schmidt, 2009) and response-time distributions (Panis \& Schmidt, 2016). In particular, these studies demonstrated that the first responses are exclusively triggered by prime properties, independent of the target, whereas only later responses are influenced by target properties.

Schmidt, Niehaus, and Nagel (2006) hence proposed a chase theory of response priming in which they formulated the chase criteria of such a feedforward system: (1) Prime rather than target signals determine the onset and initial direction of the response; (2) target signals influence the response before it is completed; (3) movement kinematics initially depend on prime characteristics only and are independent of all target characteristics (see Schmidt, 2014, for precise definitions of criteria and predictions). Such a simple feedforward-sweep model seems to account very well for response priming effects at short SOAs (up to $100 \mathrm{~ms}$ ), but would predict unrealistically high error rates for longer SOAs (because in inconsistent trials, the prime would always have enough time to drive the wrong response to completion). Therefore, priming effects at longer SOAs are more plausibly carried by the content of a response buffer that carries information from both primes, but is dominated by the second one (Grainger et al., 2013). This buffer would allow participants to delay their responses, waiting out the target.

\section{Event history analysis}

The aims of the current study were to trace sequential priming effects over the time course of a trial to see (a) whether sequential primes actually initiate sequential response activation, (b) whether that sequence conforms to the chase criteria at short SOAs, and (c) how the influence of the first prime changes when the interprime interval is prolonged. In order to investigate the temporal dynamics of response activation, one 
must take the passage of time into account when analyzing behavioral output. Here, we make use of a relatively new approach to analyze reaction time data: Event history analysis (EHA: Allison, 1982, 2010; Luce, 1986; Panis \& Schmidt, 2016; Singer \& Willett, 2003). In EHA, it is assumed that for each time point since target onset in each trial of an experiment, there is a risk for the response to occur. The time after target onset is subdivided into a series of nonoverlapping and contiguous time bins indexed by $\mathrm{t}, \mathrm{t} \in\{1 \ldots n\}$, and for each time bin, the discrete-time hazard probability of response occurrence is estimated. The hazard probability $h(\mathrm{t})$ is defined as the conditional probability that a response occurs sometime within bin $\mathrm{t}$ given that no response has been emitted in previous bins: $h(\mathrm{t})=\mathrm{P}(\mathrm{T}=\mathrm{t} \mid \mathrm{T} \geq \mathrm{t})$ (Allison, 1982, 2010; Luce, 1986; Panis, Torfs, Gillebert, Wagemans, \& Humphreys, 2017; Panis \& Wagemans, 2009). The survival function $S(\mathrm{t})$ $=P(\mathrm{~T}>\mathrm{t})$ estimates the probability that no response has been emitted by the time Bin $\mathrm{t}$ is completed. In addition, $P(\mathrm{t})=P(\mathrm{~T}$ $=\mathrm{t}$ ) gives the unconditional probability that a response (no matter whether correct or incorrect) occurs within Bin $t{ }^{1}$ Since correct and incorrect response occurrences are not independent (Burle, Vidal, Tandonnet, \& Hasbroucq, 2004; Praamstra \& Seiss, 2005), we calculate the conditional accuracy $c a(\mathrm{t})=P($ correct response $\mid \mathrm{T}=\mathrm{t})$, the probability that a response emitted in time Bin $\mathrm{t}$ is correct. Together, $h(\mathrm{t})$ and $c a(t)$ give an unbiased description of the time course of the latency and accuracy of responses (Panis \& Hermens, 2014; Panis \& Schmidt, 2016).

\section{Current study}

Here, we investigate the effects of two sequential primes followed by one target on response occurrence and accuracy in a response priming experiment. Our goal was to investigate (a) whether sequential primes actually initiate sequential response activation, or integrate in a buffer before a response is emitted, (b) whether that response activation sequence conforms to the rapid-chase criteria at short SOAs, and (c) how the influence of the first prime changes when the SOAs are all prolonged.

We designed a stimulus layout where two primes can be presented in sequence without mutual interference and without masking. Further, we varied the timing of the stimuli by keeping the Prime 1 target (P1-T or SOA1) SOA fixed and moving the onset of Prime 2, resulting in different combinations of Prime 1-Prime 2 (P1-P2) and Prime 2-target (P2-T or SOA2) SOAs. Each prime could either be consistent or inconsistent to the target. In a first experiment we investigated quick successions of primes and target, a second experiment used prolonged stimulus-onset asynchronies. We reasoned from the idea that when the P1-T SOA is short (Experiment 1), participants can rely on feedforward response activation and give speeded responses without using the response buffer. In contrast, when the P1-T SOA is long (Experiment 2), participants are forced to withhold responses in order to avoid errors triggered by inconsistent primes, and in that situation the response buffer can influence the response.

\section{Experiment 1}

\section{Method}

We constructed a stimulus arrangement dubbed the 'lollipop' that allows us to present a sequence of primes and targets without any spatial overlap or masking (see Fig. 1). The lollipop consisted of a large circle subdivided into eight segments that would contain the primes. A circle in the center of the lollipop contained the target and served as fixation point. Participants were instructed to give speeded responses to the color of the target — red or green - with two successive primes appearing prior to its onset. For the first prime, every other lollipop segment briefly changed color simultaneously (all either red or green). For the second prime, the previously unoccupied segments all briefly turned red or green simultaneously, independent of the color of the first prime.

\section{Participants}

Twelve participants (seven female, ages 22-36 years, $M=$ 28.2 years) were recruited out of the pool of students of the University of Kaiserslautern. They participated in one 60minute session for each experiment and were rewarded with course credits. All of them had normal or corrected-to-normal vision (17\% with correction). Each participant gave informed consent and was treated in accordance with the ethical standards of the American Psychological Association.

\section{Apparatus and stimuli}

Participants sat comfortably on a chair in front of a 17 -inch VGA cathode-ray monitor (refresh rate of $75 \mathrm{~Hz}$, resolution of $1,280 \times 1,024)$ in a dimly lit room, such that their faces were at a distance of roughly $80 \mathrm{~cm}$ from the screen. Responses were collected with a USTC Response Time Box (Li, Liang, Kleiner, \& Lu, 2010). Microsoft Windows XP served as the operating system and the experiments were written in MATLAB, using the Psychophysics Toolbox extensions (Brainard, 1997; Kleiner et al., 2007; Pelli, 1997).

Prime and target stimuli appeared inside the lollipop frame, which was present throughout the trial (see Fig. 1). The frame was shown in white $\left(54.3 \mathrm{~cd} / \mathrm{m}^{2}\right.$, line width 2 pixels) against a black background $\left(0.03 \mathrm{~cd} / \mathrm{m}^{2}\right)$ and consisted of a central circle $\left(\varnothing 0.8 \mathrm{~cm}, 0.57^{\circ}\right)$ for the target and a larger circle (Ø 2.4 $\mathrm{cm}, 1.72^{\circ}$ ) for the primes. The large circle was subdivided into 
eight $45^{\circ}$ segments by horizontal, vertical and diagonal lines. The first prime (P1) was presented by filling-in four noncontiguous segments with the same color (either red, 11.0 $\mathrm{cd} / \mathrm{m}^{2}, x=.45, y=.30$, or green, $11.0 \mathrm{~cd} / \mathrm{m}^{2}, x=.24, y=$ $.40)$. The second prime (P2) was then presented in the remaining segments. The two sets of segments were randomly assigned to colors and primes. As a target stimulus (T), the inner small circle of the frame was filled with either red or green color.

\section{Procedure}

Experiment 1 lasted 60 minutes. The experiment started with one practice and two experimental blocks with 50 trials each in which no prime [N] was displayed. This had the purpose to accustom the participants to the procedure. After completion of this task, prime conditions were administered to the participants. Each prime could either be consistent (same color) or inconsistent (different color) with the target. There were two single-prime conditions, consistent [C] and inconsistent [I], and four double-prime conditions, consistent-consistent [CC], consistent-inconsistent [CI], inconsistent-consistent [IC], and inconsistent-inconsistent [II]. (Throughout this paper, we always code consistency relative to the target.) Again, participants had to complete one practice block, this time followed by 25 experimental blocks, with 56 trials each. Each block contained eight single-prime trials and 48 double-prime trials. Altogether, this led to participants completing 100 trials each for the no-prime, two one-prime and twelve double-prime (three $\mathrm{SOA} \times$ four prime combinations) conditions. $^{2}$

Each trial began with the onset of the lollipop frame (see Fig. 1). After $493 \mathrm{~ms}$ of fixation, P1 was presented in either red or green for $13 \mathrm{~ms}$, except for the no-prime trials during which all segments remained black (such that the SOA structure was maintained even when one or both primes were absent). After a P1-P2 SOA of 27, 53, or $80 \mathrm{~ms}$, either a red or green P2 was presented for another $13 \mathrm{~ms}$, except for the noprime and single-prime trials during which all segments stayed black. Finally, after a P2-T SOA of 80, 53, or $27 \mathrm{ms,}$ a red or green target followed. As a result, the SOA between P1 and T was always $107 \mathrm{~ms}$. The target stayed on-screen for $107 \mathrm{~ms}$. Participants were instructed to fixate the target circle at the center of the frame (see Fig. 1) and to respond to the target color as quickly and accurately by pressing one of two response buttons with their left or right index finger, while all other stimuli were irrelevant. After detection of the manual response, a feedback display was shown for $500 \mathrm{~ms}$, followed by a blank screen for $360 \mathrm{~ms}$ before the next trial started. Participants received a "too slow" feedback message if their RT was slower than 999 ms. During practice trials they received an additional "wrong" feedback message if their response was incorrect and "correct" if their response was correct. Additionally, after each block participants received feedback on their performance (percentage correct, number of errors, mean reaction time) and could take a short rest if desired. Color-to-button mapping was fixed for each participant and counterbalanced across participants. All stimulus conditions, except for the blocked no-prime condition, occurred randomly and equiprobably over the course of a session.

\section{Analysis of mean error rate and mean correct RT}

In a first step, mean reaction times (RT) and error rates (ER) were inspected. We performed two sets of analyses. First, one-

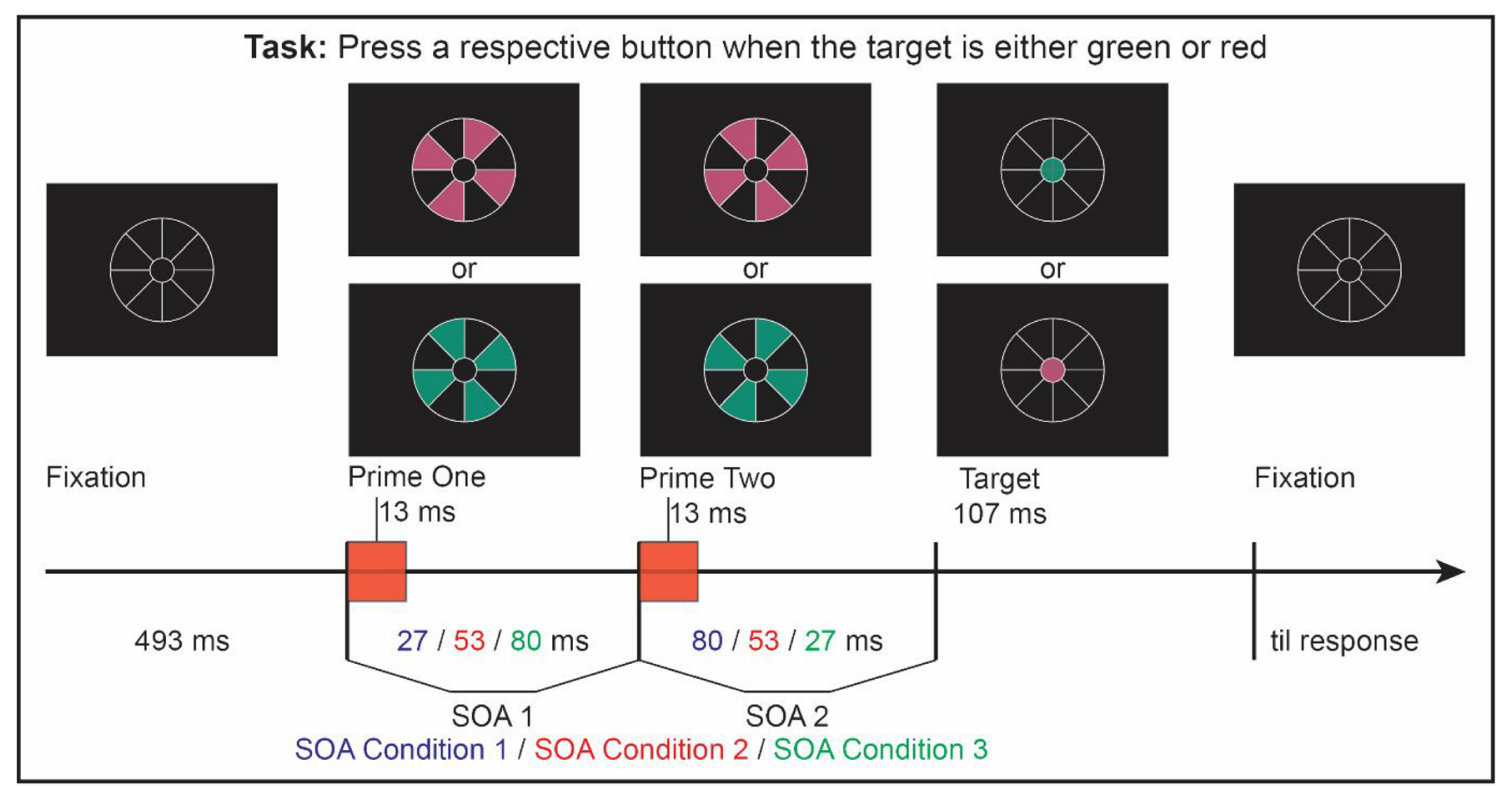

Fig. 1 Stimulus displays and design. After fixating the center of the white lollipop frame, a sequence of two primes and a target is presented, with SOA1SOA 2 combinations of $27 / 80,53 / 53$, or $80 / 27$ 
Table 1 Selected hazard model for Experiment 1

\begin{tabular}{|c|c|c|c|c|c|c|c|c|c|c|c|}
\hline \multirow{2}{*}{\multicolumn{2}{|c|}{ Effect }} & \multicolumn{2}{|c|}{$(175,200]$} & \multicolumn{4}{|c|}{$(250,275]$} & \multicolumn{2}{|c|}{$(300,325]$} & \multicolumn{2}{|c|}{$(375,400]$} \\
\hline & & PE & $p$ & $\mathrm{PE}$ & $\mathrm{SE}$ & $t$ & $p$ & $\mathrm{PE}$ & $p$ & $\mathrm{PE}$ & $p$ \\
\hline 1 & Intercept & \multirow[t]{5}{*}{-4.650} & \multirow[t]{5}{*}{$0.0000^{* * * *}$} & -2.361 & 0.260 & -9.084 & $0.0000^{* * * *}$ & \multirow[t]{5}{*}{-1.318} & \multirow[t]{5}{*}{$0.0000^{* * * *}$} & \multirow[t]{5}{*}{-0.620} & \multirow[t]{5}{*}{$0.0000^{* * * *}$} \\
\hline 2 & TIME & & & 0.637 & 0.039 & 16.504 & $0.0000^{\text {***** }}$ & & & & \\
\hline 3 & TIME $^{2}$ & & & -0.054 & 0.004 & -14.811 & $0.0000^{* * * *}$ & & & & \\
\hline 4 & TIME $^{3}$ & & & -0.003 & 0.001 & -3.114 & $0.0018^{* * *}$ & & & & \\
\hline 5 & TIME $^{4}$ & & & 0.000 & 0.000 & 3.366 & $0.0008^{* * * *}$ & & & & \\
\hline 6 & TRIAL & \multirow[t]{2}{*}{-0.003} & \multirow[t]{2}{*}{0.5553} & 0.005 & 0.003 & 1.919 & 0.0549 & \multirow[t]{2}{*}{0.011} & \multirow[t]{2}{*}{$0.0000^{* * * *}$} & \multirow[t]{2}{*}{0.019} & \multirow[t]{2}{*}{$0.0000^{* * *}$} \\
\hline 7 & TIME:TRIAL & & & 0.003 & 0.001 & 3.418 & $0.0006^{\text {***** }}$ & & & & \\
\hline 8 & $\mathrm{C}$ & \multirow[t]{4}{*}{0.179} & \multirow[t]{4}{*}{0.1651} & 0.729 & 0.055 & 13.260 & $0.0000^{* * * *}$ & \multirow[t]{4}{*}{0.549} & \multirow[t]{4}{*}{$0.0000^{* * *}$} & -0.029 & 0.6932 \\
\hline 9 & TIME:C & & & -0.006 & 0.025 & -0.252 & 0.8007 & & & & \\
\hline 10 & $\mathrm{TIME}^{2}: \mathrm{C}$ & & & -0.050 & 0.009 & -5.615 & $0.0000^{* * * *}$ & & & & \\
\hline 11 & $\mathrm{TIME}^{3}: \mathrm{C}$ & & & 0.004 & 0.002 & 2.820 & $0.0048^{* * *}$ & & & & \\
\hline 12 & I & 0.113 & 0.3951 & -0.506 & 0.070 & -7.242 & $0.0000^{* * * * *}$ & -0.688 & $0.0000^{* * * *}$ & -0.308 & $0.0000^{* * * *}$ \\
\hline 13 & TIME:I & & & -0.196 & 0.041 & -4.785 & $0.0000^{* * * *}$ & & & & \\
\hline 14 & TIME $^{2}: \mathrm{I}$ & & & 0.042 & 0.010 & 4.291 & $0.0000^{* * * *}$ & & & & \\
\hline 15 & $\mathrm{TIME}^{3}: \mathrm{I}$ & & & 0.008 & 0.003 & 2.915 & $0.0036^{* *}$ & & & & \\
\hline 16 & TIME $^{4}: \mathrm{I}$ & & & -0.001 & 0.000 & -3.736 & $0.0002^{* * * *}$ & & & & \\
\hline 17 & $\mathrm{~N}$ & 0.666 & $0.0000^{* * * *}$ & 0.476 & 0.061 & 7.775 & $0.0000^{\text {***** }}$ & 0.050 & 0.3661 & -0.355 & $0.0000^{* * * *}$ \\
\hline 18 & TIME:N & & & -0.187 & 0.024 & -7.732 & $0.0000^{\text {***** }}$ & & & & \\
\hline 19 & $\mathrm{TIME}^{2}: \mathrm{N}$ & & & -0.024 & 0.007 & -3.373 & $0.0007^{* * * * *}$ & & & & \\
\hline 20 & $\mathrm{TIME}^{3}: \mathrm{N}$ & & & 0.006 & 0.001 & 4.673 & $0.0000^{* * * *}$ & & & & \\
\hline 21 & II & 0.285 & $0.0012^{* *}$ & -0.403 & 0.054 & -7.423 & $0.0000^{* * * *}$ & -0.623 & $0.0000^{\text {***** }}$ & -0.396 & $0.0000^{* * * * *}$ \\
\hline 22 & TIME:II & & & -0.193 & 0.025 & -7.688 & $0.0000^{* * * *}$ & & & & \\
\hline 23 & TIME $^{2}:$ II & & & 0.035 & 0.006 & 5.714 & $0.0000^{* * * *}$ & & & & \\
\hline 24 & TIME $^{3}:$ II & & & 0.005 & 0.002 & 3.146 & $0.0017^{* * *}$ & & & & \\
\hline 25 & TIME $^{4}:$ II & & & -0.001 & 0.000 & -3.747 & $0.0002^{* * * *}$ & & & & \\
\hline 26 & $\mathrm{CC}$ & 0.389 & $0.0000^{\text {******* }}$ & 0.627 & 0.049 & 12.860 & $0.0000^{\text {***** }}$ & 0.407 & $0.0000^{* * * *}$ & -0.084 & 0.1254 \\
\hline 27 & TIME:CC & & & -0.054 & 0.016 & -3.404 & $0.0007^{* * * *}$ & & & & \\
\hline 28 & $\mathrm{TIME}^{2}: \mathrm{CC}$ & & & -0.034 & 0.005 & -6.597 & $0.0000^{* * * * *}$ & & & & \\
\hline 29 & $\mathrm{TIME}^{3}: \mathrm{CC}$ & & & 0.003 & 0.001 & 3.835 & $0.0001^{* * * *}$ & & & & \\
\hline 30 & $\mathrm{CI}$ & -0.256 & $0.0000^{* * * *}$ & -0.256 & 0.032 & -8.003 & $0.0000^{* * * * *}$ & -0.256 & $0.0000^{\text {***** }}$ & -0.256 & $0.0000^{* * * *}$ \\
\hline 31 & SOA_53_53 & -0.191 & $0.0000^{\text {****** }}$ & -0.191 & 0.032 & -5.985 & $0.0000^{* * * *}$ & -0.191 & $0.0000^{* * * *}$ & -0.191 & $0.0000^{* * * * *}$ \\
\hline 32 & SOA_80_27 & -0.223 & $0.0027^{* *}$ & -0.327 & 0.046 & -7.053 & $0.0000^{* * * *}$ & -0.336 & $0.0000^{* * * *}$ & -0.261 & $0.0000^{* * * *}$ \\
\hline 33 & TIME:SOA_80_27 & & & -0.017 & 0.012 & -1.359 & 0.1742 & & & & \\
\hline 34 & TIME $^{2}:$ SOA_80_27 & & & 0.006 & 0.002 & 2.585 & $0.0097^{* * *}$ & & & & \\
\hline 35 & II:SOA_53_53 & 0.124 & $0.0267^{*}$ & 0.124 & 0.056 & 2.216 & $0.0267^{*}$ & 0.124 & $0.0267^{*}$ & 0.124 & $0.0267^{*}$ \\
\hline 36 & II:SOA_80_27 & 0.245 & $0.0001^{\text {****** }}$ & 0.245 & 0.062 & 3.928 & $0.0001^{\text {***** }}$ & 0.245 & $0.0001^{* * * *}$ & 0.245 & $0.0001^{* * * * *}$ \\
\hline 37 & CC:SOA_53_53 & 0.167 & $0.0021^{* * *}$ & 0.167 & 0.054 & 3.078 & $0.0021^{* *}$ & 0.167 & $0.0021^{* *}$ & 0.167 & $0.0021^{* *}$ \\
\hline 38 & CC:SOA_80_27 & 0.287 & $0.0000^{* * * *}$ & 0.287 & 0.061 & 4.693 & $0.0000^{* * * * *}$ & 0.287 & $0.0000^{* * * *}$ & 0.287 & $0.0000^{* * *}$ \\
\hline 39 & CI:SOA_80_27 & 0.212 & $0.0001^{* * * *}$ & 0.212 & 0.055 & 3.845 & $0.0001^{* * * * *}$ & 0.212 & $0.0001^{* * * *}$ & 0.212 & $0.0001^{\text {**** }}$ \\
\hline & $S D$ Intercept & 1.122 & & .892 & & & & .679 & & .431 & \\
\hline & $S D$ TIME & .124 & & .124 & & & & .124 & & .123 & \\
\hline & Correlation & -.937 & & -.899 & & & & -.818 & & -.427 & \\
\hline
\end{tabular}

Note. Parameter estimates (PE) and test statistics. During model selection, TIME was centered on bin 275. The selected model was refitted three times with TIME centered on bin 200,325 , and 400 , respectively. $S D=$ standard deviation

way repeated-measures ANOVAs, with the factor consistency (consistent, inconsistent, no prime), were performed for single-prime and no-prime conditions, one for each of the two dependent variables, RT and ER. A total of 3,600 trials 
Table 2 Selected $c a(\mathrm{t})$ model for Experiment 1. Parameter estimates (PE) and test statistics

\begin{tabular}{|c|c|c|c|c|c|c|c|c|c|c|c|}
\hline \multirow{2}{*}{\multicolumn{2}{|c|}{ Effect }} & \multicolumn{2}{|c|}{$(150,175]$} & \multicolumn{2}{|c|}{$(200,225]$} & \multicolumn{4}{|c|}{$(250,275]$} & \multicolumn{2}{|c|}{$(375,400]$} \\
\hline & & PE & $p$ & $\mathrm{PE}$ & $p$ & PE & $\mathrm{SE}$ & $t$ & $p$ & PE & $p$ \\
\hline 1 & Intercept & -2.379 & $0.0000^{* * * *}$ & -0.076 & 0.7026 & 1.918 & 0.157 & 12.187 & $0.0000^{* * * *}$ & 3.229 & $0.0000^{* * * *}$ \\
\hline 2 & TIME & & & & & 0.797 & 0.062 & 12.806 & $0.0000^{* * * *}$ & & \\
\hline 3 & TIME $^{2}$ & & & & & -0.118 & 0.015 & -7.696 & $0.0000^{* * * *}$ & & \\
\hline 4 & TIME $^{3}$ & & & & & -0.006 & 0.003 & -1.993 & $0.0463^{*}$ & & \\
\hline 5 & TIME $^{4}$ & & & & & 0.002 & 0.000 & 4.087 & $0.0000^{* * * *}$ & & \\
\hline 6 & $\mathrm{C}$ & 5.213 & $0.0000^{* * * *}$ & 2.906 & $0.0000^{* * * * *}$ & 1.339 & 0.232 & 5.764 & $0.0000^{* * * *}$ & 0.659 & 0.1446 \\
\hline 7 & TIME:C & & & & & -0.599 & 0.109 & -5.516 & $0.0000^{* * * *}$ & & \\
\hline 8 & $\mathrm{TIME}^{2}: \mathrm{C}$ & & & & & 0.093 & 0.027 & 3.369 & $0.0008^{* * * *}$ & & \\
\hline 9 & I & 0.407 & 0.5240 & -2.040 & $0.0000^{* * * *}$ & -2.405 & 0.188 & -12.783 & $0.0000^{* * * *}$ & -0.126 & 0.6165 \\
\hline 10 & TIME:I & & & & & 0.188 & 0.088 & 2.139 & $0.0324^{*}$ & & \\
\hline 11 & TIME $^{2}: \mathrm{I}$ & & & & & 0.148 & 0.025 & 5.903 & $0.0000^{* * * *}$ & & \\
\hline 12 & TIME $^{3}: \mathrm{I}$ & & & & & -0.019 & 0.004 & -4.559 & $0.0000^{* * * *}$ & & \\
\hline 13 & $\mathrm{~N}$ & 3.456 & $0.0000^{* * * *}$ & 1.421 & $0.0000^{* * * *}$ & 0.112 & 0.178 & 0.629 & 0.5292 & 0.013 & 0.9667 \\
\hline 14 & TIME:N & & & & & -0.473 & 0.072 & -6.584 & $0.0000^{* * * *}$ & & \\
\hline 15 & $\mathrm{TIME}^{2}: \mathrm{N}$ & & & & & 0.091 & 0.018 & 5.130 & $0.0000^{* * * * *}$ & & \\
\hline 16 & II & -0.900 & 0.1208 & -2.376 & $0.0000^{* * * *}$ & -2.438 & 0.146 & -16.694 & $0.0000^{* * * *}$ & -0.139 & 0.4832 \\
\hline 17 & TIME:II & & & & & 0.227 & 0.073 & 3.108 & $0.0019^{* * *}$ & & \\
\hline 18 & TIME $^{2}:$ II & & & & & 0.106 & 0.021 & 4.951 & $0.0000^{\text {***** }}$ & & \\
\hline 19 & $\mathrm{TIME}^{3}:$ II & & & & & -0.012 & 0.003 & -3.655 & $0.0003^{\text {****** }}$ & & \\
\hline 20 & $\mathrm{CC}$ & 5.349 & $0.0000^{* * * *}$ & 3.189 & $0.0000^{* * * *}$ & 1.624 & 0.175 & 9.269 & $0.0000^{* * * *}$ & 0.309 & 0.2298 \\
\hline 21 & TIME:CC & & & & & -0.634 & 0.074 & -8.596 & $0.0000^{* * * * *}$ & & \\
\hline 22 & $\mathrm{TIME}^{2}: \mathrm{CC}$ & & & & & 0.074 & 0.016 & 4.659 & $0.0000^{\text {***** }}$ & & \\
\hline 23 & $\mathrm{CI}$ & 4.742 & $0.0000^{* * * *}$ & 0.487 & 0.0502 & -1.440 & 0.164 & -8.805 & $0.0000^{* * * * *}$ & -0.471 & 0.0513 \\
\hline 24 & TIME:CI & & & & & -0.494 & 0.070 & -7.041 & $0.0000^{* * * *}$ & & \\
\hline 25 & $\mathrm{TIME}^{2}: \mathrm{CI}$ & & & & & 0.207 & 0.024 & 8.478 & $0.0000^{\text {***** }}$ & & \\
\hline 26 & $\mathrm{TIME}^{3}: \mathrm{CI}$ & & & & & -0.014 & 0.004 & -3.927 & $0.0001^{* * * *}$ & & \\
\hline 27 & SOA_53_53 & -0.461 & 0.0553 & -0.391 & $0.0226^{*}$ & -0.320 & 0.115 & -2.780 & $0.0054^{* *}$ & -0.144 & 0.3858 \\
\hline 28 & TIME:SOA_53_53 & & & & & 0.035 & 0.039 & 0.893 & 0.3717 & & \\
\hline 29 & SOA_80_27 & -1.177 & $0.0179^{*}$ & -1.246 & $0.0000^{* * * *}$ & -1.137 & 0.168 & -6.750 & $0.0000^{* * * * *}$ & -0.091 & 0.7183 \\
\hline 30 & TIME:SOA_80_27 & & & & & 0.099 & 0.075 & 1.322 & 0.1862 & & \\
\hline 31 & TIME $^{2}:$ SOA_80_27 & & & & & 0.022 & 0.013 & 1.736 & 0.0826 & & \\
\hline 32 & II:SOA_80_27 & -0.367 & 0.6955 & 0.626 & 0.1782 & 1.074 & 0.234 & 4.595 & $0.0000^{\text {****** }}$ & -0.190 & 0.5370 \\
\hline 33 & TIME:II:SOA_80_27 & & & & & 0.088 & 0.133 & 0.661 & 0.5085 & & \\
\hline 34 & TIME $^{2}:$ II:SOA_80_27 & & & & & -0.068 & 0.023 & -2.928 & $0.0034^{* * *}$ & & \\
\hline 35 & CC:SOA_80_27 & 2.505 & $0.0002^{* * * *}$ & 1.928 & $0.0001^{* * * *}$ & 1.351 & 0.322 & 4.199 & $0.0000^{* * * *}$ & -0.092 & 0.8261 \\
\hline 36 & $\begin{array}{l}\text { TIME:CC:SOA_80_ } \\
27\end{array}$ & & & & & -0.289 & 0.105 & -2.750 & $0.0060^{* * *}$ & & \\
\hline 37 & CI:SOA_53_53 & 1.327 & $0.0015^{* *}$ & 0.957 & $0.0013^{* *}$ & 0.587 & 0.195 & 3.012 & $0.0026^{* * *}$ & -0.339 & 0.2108 \\
\hline 38 & TIME:CI:SOA_53_53 & & & & & -0.185 & 0.068 & -2.733 & $0.0063^{* * *}$ & & \\
\hline 39 & CI:SOA_80_27 & 4.495 & $0.0000^{* * * *}$ & 3.388 & $0.0000^{* * * *}$ & 2.281 & 0.256 & 8.911 & $0.0000^{* * * *}$ & -0.486 & 0.1392 \\
\hline \multirow[t]{4}{*}{40} & TIME:CI:SOA_80_27 & & & & & -0.553 & 0.085 & -6.521 & $0.0000^{* * * *}$ & & \\
\hline & $S D$ Intercept & .373 & & .324 & & .353 & & & & .353 & \\
\hline & $S D$ TIME & .081 & & .081 & & .081 & & & & .081 & \\
\hline & Correlation & -.496 & & -.068 & & .398 & & & & .406 & \\
\hline
\end{tabular}

Note. During model selection TIME was centered on bin 275 . The selected model was refitted three times with TIME centered on bin 175,225 , and 400 , respectively 


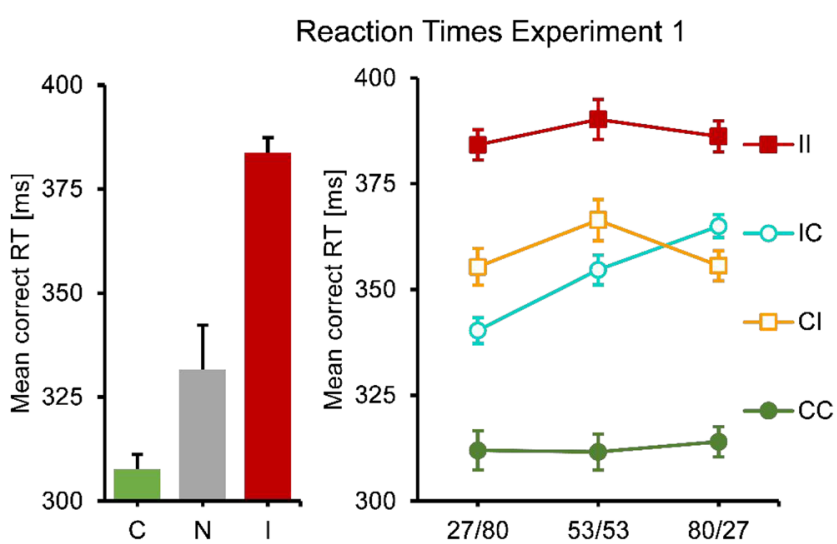

Fig. 2 Mean correct RT results for Experiment 1. No and single-prime conditions: Left panel, error bars resemble the standard error of the mean, consistency conditions on the $x$-axes. Double-prime conditions: Right panel, error bars resemble the standard error of the mean, separate lines for consistency conditions, SOA conditions on the $x$-axes

were initially available for analysis. Trials with reaction times faster than $100 \mathrm{~ms}$ or slower than $999 \mathrm{~ms}(0.5 \%)$ were excluded from the analysis. Further, error trials $(10.92 \%)$ were excluded from RT analysis.

Second, two $3(\mathrm{SOA}) \times 4$ (consistency) repeated-measures ANOVAs were performed for all double-prime conditions, one each for RT and ER. A total of 14,400 trials were initially available for analysis. Trials with reaction times faster than $100 \mathrm{~ms}$ or slower than $999 \mathrm{~ms}(0.53 \%)$ were excluded from the analysis. In addition, error trials $(13.53 \%)$ were excluded from RT analysis. To follow up significant interaction effects, one-way repeated-measures ANOVAs, with the four-level factor consistency (CC, CI, IC, II) were performed separately for each SOA condition. Greenhouse-Geisser-corrected $p$ values were used. To satisfy ANOVA requirements error rates were arcsine transformed. Additional within-subjects contrasts were calculated to further investigate significant main effects.

\section{Error Rates Experiment 1}

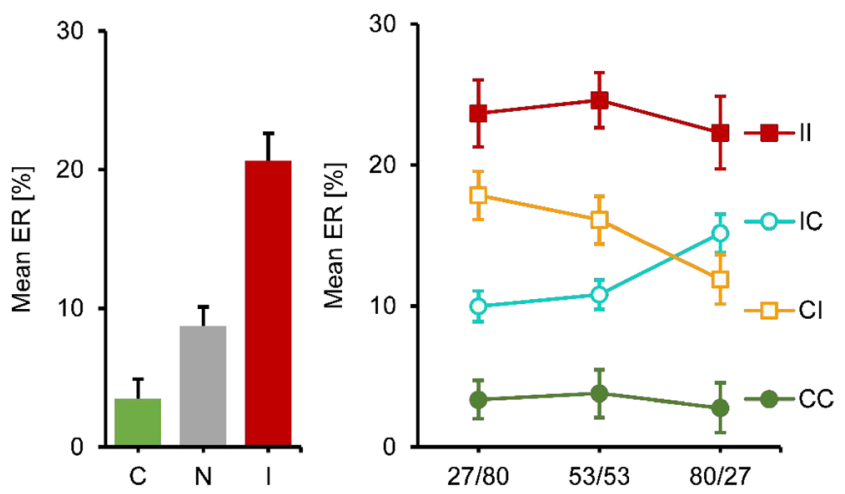

Fig. 3 Mean ER results for Experiment 1. No and single-prime conditions: Left panel, error bars resemble the standard error of the mean, consistency conditions on the $x$-axes. Double-prime conditions: Right panel, error bars resemble the standard error of the mean, separate lines for consistency conditions, SOA conditions on the $x$-axes

\section{Event history analysis}

Sample-based descriptive estimates of hazard function $h(\mathrm{t})$, survival function $S(t)$, probability function $P(\mathrm{t})$, and conditional-accuracy function $c a(\mathrm{t})$ were calculated for each combination of condition. For the purpose of visually inspecting the descriptive functions data was pooled across participants to reduce noise, after checking that each participant showed similarly timed effects. A censoring time of $600 \mathrm{~ms}$ was used because only a limited amount of responses occurred afterwards. To provide a high temporal resolution and still obtain stable estimates a bin size of $25 \mathrm{~ms}$ was used. In other words, the first $600 \mathrm{~ms}$ after target onset were divided into 24 time bins of $25 \mathrm{~ms}$ indexed by $\mathrm{t}=1$ to 24 . Trials with RTs longer than $600 \mathrm{~ms}$ were treated as right-censored observations. Time bins are denoted by the endpoint of the interval they span, such that Bin $11=$ Bin $275=(250,275]$.

Next, discrete-time hazard models and conditional accuracy models were estimated by computing linear mixed-effects regression models in $R$ (R Core Team, 2014; function glmmPQL ${ }^{3}$ of package MASS; see also Panis \& Schmidt, 2016). For the hazard models we used the complementary $\log$-log (cloglog) link. ${ }^{4}$ An example discrete-time hazard model with three predictors can be written as follows: $\operatorname{clog} \log [h(\mathrm{t})]=\ln (-\ln [1-h(\mathrm{t})])=\left[\alpha_{0} \mathrm{ONE}+\alpha_{1}(\right.$ TIME -1$)$ $\left.+\alpha_{2}(\text { TIME }-1)^{2}+\alpha_{3}(\text { TIME }-1)^{3}\right]+\left[\beta_{1} X_{1}+\beta_{2} X_{2}+\right.$ $\beta_{3} X_{2}$ (TIME -1$\left.)\right]$. The main predictor variable TIME is the time bin index $t$ which is centered on value 1 in this example. The first set of terms within brackets, the alpha parameters multiplied by their polynomial specifications of (centered) time, represents the shape of the baseline cloglog-hazard function (i.e., when all predictors $X_{i}$ take on a value of zero). The second set of terms (the beta parameters) represents the vertical shift in the baseline cloglog-hazard for a 1 unit increase in the respective predictor. For example, the effect of a 1 unit increase in $X_{1}$ is to vertically shift the whole baseline clogloghazard function with $\beta_{1}$ cloglog-hazard units. However, if the predictor interacts linearly with time (see $\mathrm{X}_{2}$ in the example), then the effect of a 1 unit increase in $X_{2}$ is to vertically shift the predicted cloglog-hazard in Bin 1 with $\beta_{2}$ cloglog-hazard units (when TIME $-1=0$ ), in Bin 2 with $\beta_{2}+\beta_{3}$ clogloghazard units (when TIME $-1=1$ ), and so forth. To interpret the effects of the predictors, the parameter estimates are antilogged, resulting in a hazard ratio.

For our data we centered TIME on Bin 275 during model selection. TRIAL number was included as a predictor (centered on Trial 1,000 , rescaled by dividing by 100 ), in order to account for across-trial learning effects in $h(\mathrm{t})$. The intercept and the linear effect of TIME were treated as random effects to deal with the correlated data resulting from the repeated measures on the same subjects. ${ }^{5}$ The IC-27/80 condition (P1: inconsistent, P2: consistent, SOA1: $27 \mathrm{~ms}, \mathrm{SOA} 2: 80 \mathrm{~ms}$ ) was 

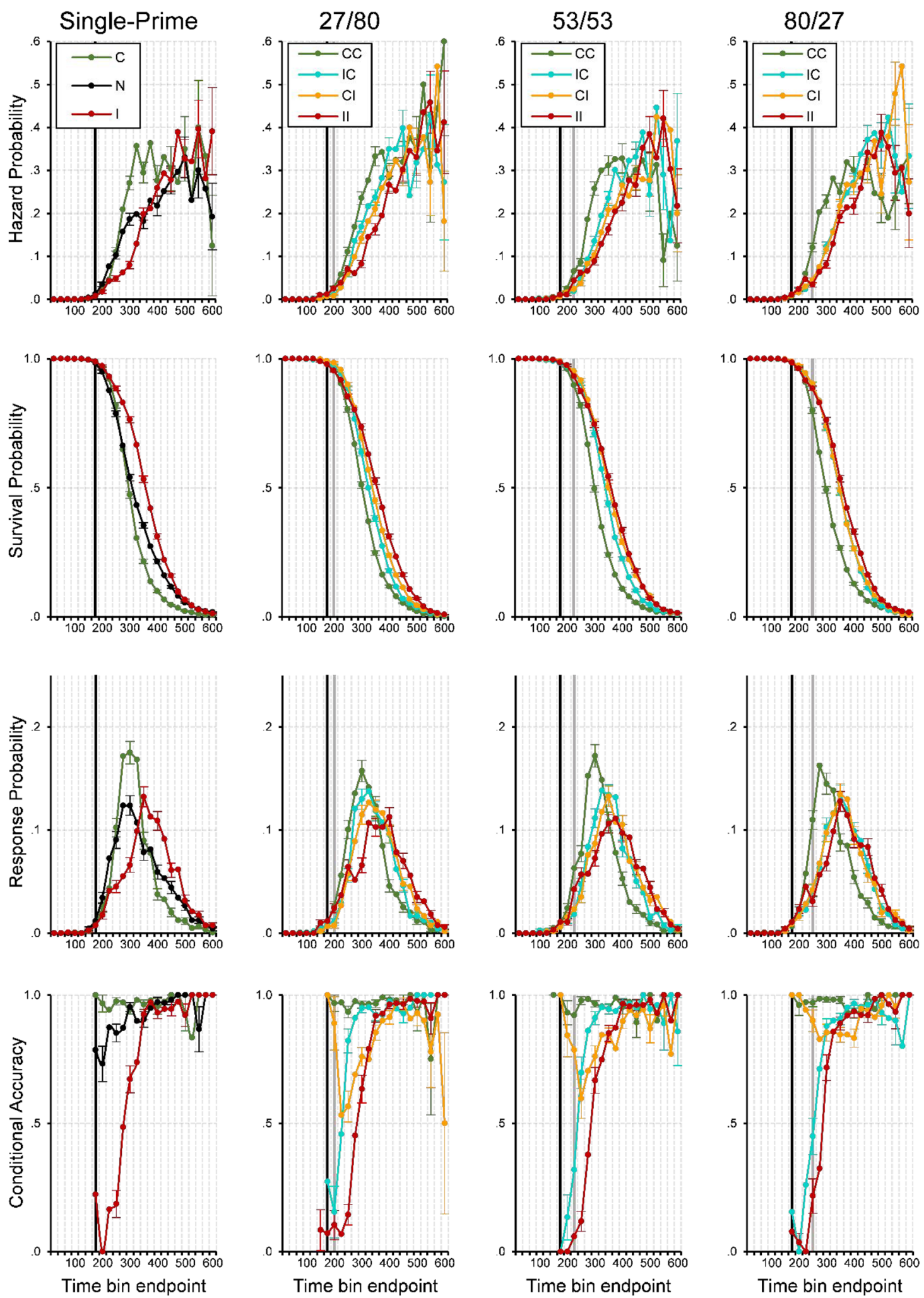

chosen as a baseline condition. Because TIME and TRIAL are centered, the intercept of the hazard regression model refers to Bin 275 in Trial 1,000 of the IC-27/80 condition.
To estimate the parameters of an $h(\mathrm{t})$ model, we must create a dataset where each row corresponds to a time bin of a trial of a participant (a subject-trial-bin oriented data set). 
Fig. 4 Sample-based estimates of $h(\mathrm{t}), S(\mathrm{t}), P(\mathrm{t})$, and $c a(\mathrm{t})$ aggregated across all participants in Experiment 1, for the first 24 bins (or $600 \mathrm{~ms}$ ) after target onset. Bin width equals $25 \mathrm{~ms}$. First column: Black lines represent the no-prime condition, green lines the consistent single-prime condition, and red lines the inconsistent single-prime condition. Second to last column: Each column represents a different SOA condition. Green lines represent consistent-consistent conditions, cyan lines inconsistentconsistent conditions, orange lines consistent-inconsistent conditions, red lines inconsistent-inconsistent conditions. Black vertical lines highlight bins at $\sim 250-275 \mathrm{~ms}$ after onset of P1, grey vertical lines after onset of P2. Note that we only plotted a $c a(\mathrm{t})$ estimate if the corresponding hazard for that bin was larger than .005 . For better visibility only every second error bar is depicted

Specifically, each time bin that was at risk for event occurrence in a trial was scored on the dependent variable EVENT $(0=$ no response occurred $; 1=$ response occurred $)$, the centered covariates TIME, TRIAL, the variable SUBJECT, and the dummy-coded dichotomous experimental predictor variables (C, I, N, II, CC, CI, SOA_53_53, SOA_80_27). Only the time range between 125 and $450 \mathrm{~ms}$ was modeled, because most responses occurred in this range. Trials with RTs longer than $450 \mathrm{~ms}$ were treated as right-censored observations, and trials with RT smaller or equal to $125 \mathrm{~ms}$ were discarded. The expanded (subject-trial-bin oriented) data set contained 157,656 rows.

For $c a(\mathrm{t})$ modeling, the original dataset was used where each row corresponds to one trial of one participant $(1,500 \times$ $12=18,000$ trials). We used the same model but applied the logit $\operatorname{link}^{6}$, and included only those trials with an observed response between 125 and $450 \mathrm{~ms}$ in the data set. In other words, trials with RT shorter than $125 \mathrm{~ms}$ and longer than $450 \mathrm{~ms}$ were discarded ( $11.63 \%$ of the 18,000 trials).

For both models, we started with a full model containing all fixed effects of interest (main and interaction effects of the dichotomous predictors), and their interactions with TIME (linear, quadratic, cubic, and quartic). In a step-by-step backward selection procedure, this full model was reduced to the final, selected model. More precisely, in each iteration, the effect with the largest $p>.05$ that was not part of any higher order effect left the model before the next fit. Finally, after model selection, we refitted the selected model a number of times with TIME centered each time on another bin, to see explicitly what values the parameter estimates take on according to the final model in these other bins, and whether they represent a significant effect (see Tables 1 and 2). This way, it becomes more explicit what the interaction effects including TIME imply, because we are able to study the effect of the various predictor variables at different time points.

\section{Predictions}

We expected primes to have sequential effects that are traceable over time in the conditional accuracy functions. Because
P1-T SOAs in Experiment 1 are short, the sequence of response activations should conform to the chase criteria, so that the earliest responses are controlled exclusively by the first prime, while later responses are consecutively controlled by the second prime, and the slowest responses by the target. The earliest responses should therefore be correct whenever P1 is consistent with the target and incorrect whenever it is inconsistent. In contrast, the slowest responses should be controlled mainly by the target and thus all be correct. Intermediate responses should be influenced by the second prime. From previous data, we expected that the second prime would dominate the response at the shortest P1-P2 SOA (i.e., the longest P2-T SOA), and this dominance of the second prime should decrease with increasing $\mathrm{P} 1-\mathrm{P} 2$ SOA because the first prime has progressively more time to activate a response before the second prime occurs, while the second prime has progressively less time before the target occurs (Grainger et al., 2013).

\section{Results}

\section{Analysis of mean error rate and mean correct RT}

An analysis of the single prime conditions showed that responses were faster and more accurate when primes were consistent rather than inconsistent, with the no-prime condition in between (see Figs. 2 and 3, left panel). One-way repeated-measures ANOVAs showed significant differences in RT, $F(1.45,15.90)=33.39, p<.001$, as well as error rates, $F(1.84,20.25)=35.56, p<.001$. In RTs as well as error rates, all means were significantly different from each other, all $p \leq$ .001 , except for the RT difference between consistent and noprime conditions $(p=.061)$.

In a next step, double-prime conditions were analyzed. RTs and error rates showed a similar overall pattern: Responses were fastest and most accurate for two consistent primes, slowest and least accurate for two inconsistent primes, and in between when primes were mixed (conditions CI and IC). In RTs, a two-way repeated-measures ANOVA showed a significant main effect of consistency (with levels CC, CI, IC, II), $F(2.09,22.98)=64.94, \mathrm{p}<$ .001 , a significant main effect of SOA $F(1.85,20.35)=$ $10.43, p=.001$, and a significant interaction, $F(4.00$, $44.04)=7.86, p<.001$ (see Fig. 2, right panel). This pattern was broken down into two separate ANOVAs, one for identical (CC, II) and one for different primes (CI, IC). The first one (CC versus II) only showed a significant main effect of consistency, $F(1.00,11.00)=113.30, p<.001$. This effect was constant across SOA, with no main effect of SOA or an interaction. The second test (CI versus IC) showed that RT increased with SOA, $F(1.78,19.32)=27.76, p<.001$. There was no main effect of consistency, but a significant interaction, $F(1.46,16.10)=12.69, p=.001$. IC was faster 

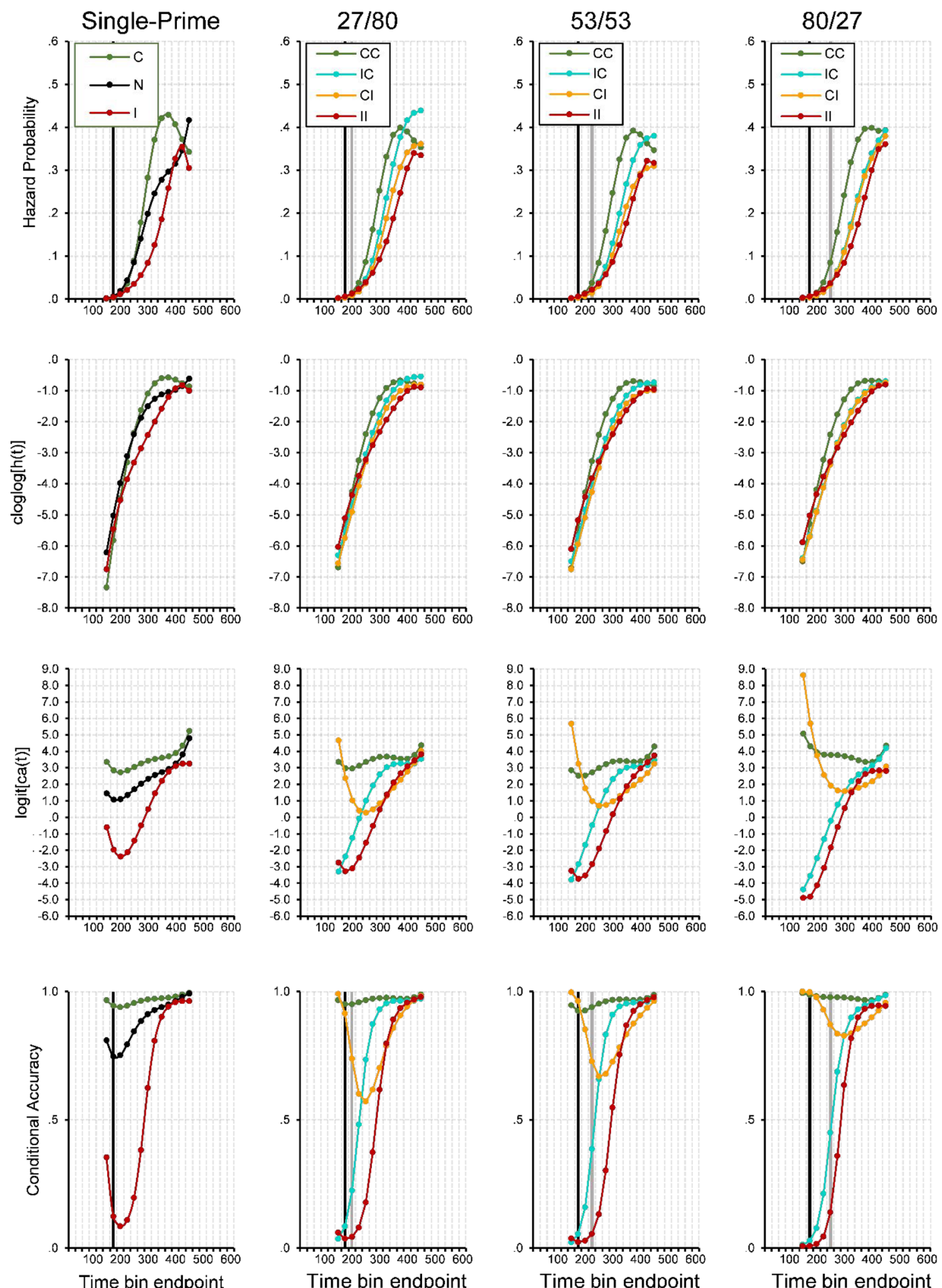

Fig. 5 Model predictions. Predicted hazard (first row), $\operatorname{cog} \log [h(\mathrm{t})]$ (second row), logit $[c a(\mathrm{t})]$ (third row), and conditional accuracy functions (fourth row) for trial 1,000 of Experiment 1. Again, black vertical lines highlight bins at $\sim 250-275 \mathrm{~ms}$ after onset of P1, grey vertical lines after onset of P2

than CI when the first SOA was short, but slower when it was long.
This pattern was even clearer (and almost perfectly symmetrical) in the error rates. An ANOVA of all double-prime 
conditions showed no main effect of SOA, but a significant main effect of consistency, $F(1.73,19.07)=37.84, p<.001$, and a significant interaction $F(3.38,37.18)=5.61, p=.002$. The follow-up analysis of CC versus II conditions only showed a main effect of consistency, $F(1.00,11.00)=59.40$, $p<.001$, but no SOA or interaction effects. The follow-up analysis of CI versus IC conditions only showed an interaction, $F(1.98,21.74)=13.87, p<.001$, but no main effects: IC was more accurate than CI when the first SOA was short, but less accurate when it was long.

\section{Event history analysis: Descriptive statistics}

In the single-prime conditions (see the first column in Fig. 4), the fastest responses occurred around $150 \mathrm{~ms}$ after target onset. Thereafter, we saw a steady increase in response hazards, which was delayed for inconsistent compared with consistent primes. This led to a marked priming effect in $h(\mathrm{t})$ of about $150 \mathrm{~ms}$ duration, and also in median RT (i.e., when the survivor function crosses .5) and mean RT. When most responses had occurred and the survival probability was low, response hazard was still at a high constant level. Strikingly, early responses were virtually always correct whenever the prime was consistent, but incorrect whenever it was inconsistent, showing that responses were exclusively determined by the prime, not the target. In inconsistent trials, conditional accuracy then quickly increased from almost zero to almost one, showing how the target took control over the response. ${ }^{7}$

Let us now take a look at the double-prime conditions where the P1-P2 SOA was short and the P2-T SOA was long (27/80' see the second column in Fig. 4), so that the impact of the second prime should be high relative to the first prime. Again, the fastest responses occurred around the same time in all priming conditions, around $150 \mathrm{~ms}$ after target onset or about $250 \mathrm{~ms}$ after P1 onset. However, initial response hazards in CI and IC conditions were lower than in CC and II conditions around 150-200 ms. This likely reflected early response competition due to conflicting prime information, as both primes activated opposite responses. After about $250 \mathrm{~ms}$ without response occurrence, the hazard functions began to differentiate and followed the order observed in mean RTs: CC was fastest, followed by IC, CI, II. This was evident in the hazard, survivor, and probability mass functions. The most diagnostic information, however, was in the conditional accuracy functions. Not surprisingly, the earliest responses were virtually all correct when both primes were consistent and all incorrect when both primes were inconsistent, which again showed that the first prime determined the earliest responses. In the II condition, conditional accuracy then quickly increased as the target took control over the response. This also occurred in the IC condition, demonstrating that the first prime alone controlled the earliest responses; but the following increase in accuracy occurred earlier than in the
II condition, demonstrating that the consistent second prime influenced the response as well. Exactly the reverse process occurred in the CI condition. Here, response accuracy was nearly perfect at first because of the consistent first prime, then decreased as the inconsistent second prime became effective, and then increased again as the target finally took control over the response.

What happened when the SOAs shifted first to $53 / 53$ and then to $80 / 27$ ? The overall pattern in $c a(\mathrm{t})$ remained the same: $\mathrm{CC}$ was always fastest with near-perfect accuracy, II was always slowest with conditional accuracy rising from very low to very high values, IC always showed an earlier increase in conditional accuracy, time-locked to the second prime's appearance. The most important change occurred in the $\mathrm{CI}$ condition. As the P1-P2 SOA became larger and the P2-T SOA became correspondingly shorter, the influence of the inconsistent second prime diminished, and the temporary drop in conditional accuracy became smaller. As with the effects observed in IC, this nadir in conditional accuracy was time locked to the second prime's presentation.

\section{Event history analysis: Inferential statistics}

To see whether these observed differences are significant we fitted hazard and conditional accuracy models to the aggregated data. Table 1 shows the selected hazard model, and Table 2 the selected $c a(\mathrm{t})$ model. Figure 5 shows predicted (i.e., model-based) hazard cloglog[h(t)], logit [ca(t)], and conditional accuracy functions for Trial 1,000 (note that choosing another trial number would not change the priming effects because we did not include interaction effects including TRIAL). The first five parameters in Table 1 model the shape of the $\operatorname{cog} \log [h(\mathrm{t})]$ function in the baseline condition, IC-27/80 in Trial 1,000 (see Fig. 5, row 2, column 2, blue line). The intercept of -2.361 cloglog-hazard units corresponds to an estimated hazard of .09 in Bin 275. This intercept increases over time in a linear, quadratic, cubic and quartic fashion (see the Parameters 2 to 5 in Table 1), so that the intercept changes from -4.65 in Bin 200 to -.62 in Bin 400 (see row 1 in Table 1).

Most importantly, compared with condition IC, changing to $\mathrm{CC}$ increases the estimated cloglog-hazard in Bin 275 by .627 units (Parameter 26), changing to CI decreases it by .256 units (Parameter 30), and changing to II decreases it by .403 units (Parameter 21; all $p<.0001$ ). While the main effects of CC and II in Bin 275 change in magnitude over time (parameter estimates in rows 27-29, 22-25), the effect of CI is time invariant. For example, note that in Bin 200 conditions, II and CC have positive parameter estimates that significantly differ from condition IC (see the parameter estimates in rows 21 and 26 in Table 1, column 3). This means that the hazard of response occurrence is lower in Bin 200 in mixed prime conditions. 
The effect of changing the SOA combination from $27 / 80$ to $53 / 53$ is to decrease the estimated cloglog-hazard by. 191 units in all bins (Parameter 31). The estimated cloglog-hazard decreases even further when SOA combination is changed to 80/ 27 (Parameters 32-34). In other words, response occurrence slows down with decreasing P2-T SOA for condition IC. However, this effect is much smaller or absent for CC and II due to interactions with 53/53 (Parameters 35 and 37) and 80/ 27 (Parameters 36 and 38). Furthermore, with SOA combination $80 / 27$ the difference between CI and IC is gone (due to Parameter 39 neutralizing the effect of Parameter 30). Finally, the hazard model also shows a significant effect of TRIAL on the estimated cloglog-hazard in bins after $275 \mathrm{~ms}$ after target onset.

The first five parameters in Table 2 model the shape of the $\operatorname{logit}[c a(\mathrm{t})]$ function in the baseline condition, IC27/80 in Trial 1,000 (see Fig. 5, row 3, column 2, blue line). The intercept of 1.918 corresponds to an estimated $c a(\mathrm{t})$ of .87 in Bin 275. This intercept increases over time in a linear, quadratic, cubic and quartic fashion (Parameters 2-5).

Most importantly, compared with condition IC, changing to $\mathrm{CC}$ increases the estimated logit-ca(t) in Bin 275 by 1.624 units (Parameter 20), changing to $\mathrm{CI}$ decreases it by 1.44 units (Parameter 23), and changing to II decreases it by 2.438 units (Parameter 16; all $p \mathrm{~s}<.0001$ ). The main effects of CC, CI, and II in Bin 275 change over time (Parameters 16-26), so that relative to IC, the positive effect of CC decreases over time, the negative effect of II first increases and then decreases, and the effect of CI shifts from positive to negative to zero. For example, note that in Bin 175 conditions $\mathrm{CC}$ and $\mathrm{CI}$ have positive parameter estimates that significantly differ from condition IC while II is not significantly different (compare rows 20 and 23 with row 16 in Table 2, column 3). This means that the conditional accuracy of these early responses is almost zero for II and IC, and almost one for CI and CC, thus reflecting first prime identity (see Fig. 5, row 4).

Increasing the P1-P2 SOA leads to a decrease in the estimated logit- $c a(\mathrm{t})$ in each bin (Parameters 27-31). Confirming the change in the temporary drop in conditional accuracy for condition $\mathrm{CI}$ in Fig. 4 are the (early and positive) interactions between CI, SOA combination, and TIME (Parameters 37-40).

\section{Summary}

As expected, mean RT and mean ER analyses of the singleprime and no-prime conditions revealed that the stimulus-set used was sufficient to produce the common finding in response priming experiments: faster and more accurate responses in consistent trials and slower and less accurate responses in inconsistent trials. Similarly, when two primes were presented, responses were fastest and most accurate for two consistent primes, slowest and least accurate for two inconsistent primes, and in between when primes were mixed. The event history analysis showed that sequential primes in fact initiate sequential response activation: (1) earliest responses were controlled exclusively by the first prime, (2) intermediate responses reflected competition between the primes where the identity of the second prime increasingly dominated the response as P2-T SOA increased, (3) this latter effect was tracking the onset of the second prime, both in magnitude and timing, and (4) only the slowest responses were clearly controlled by the target.

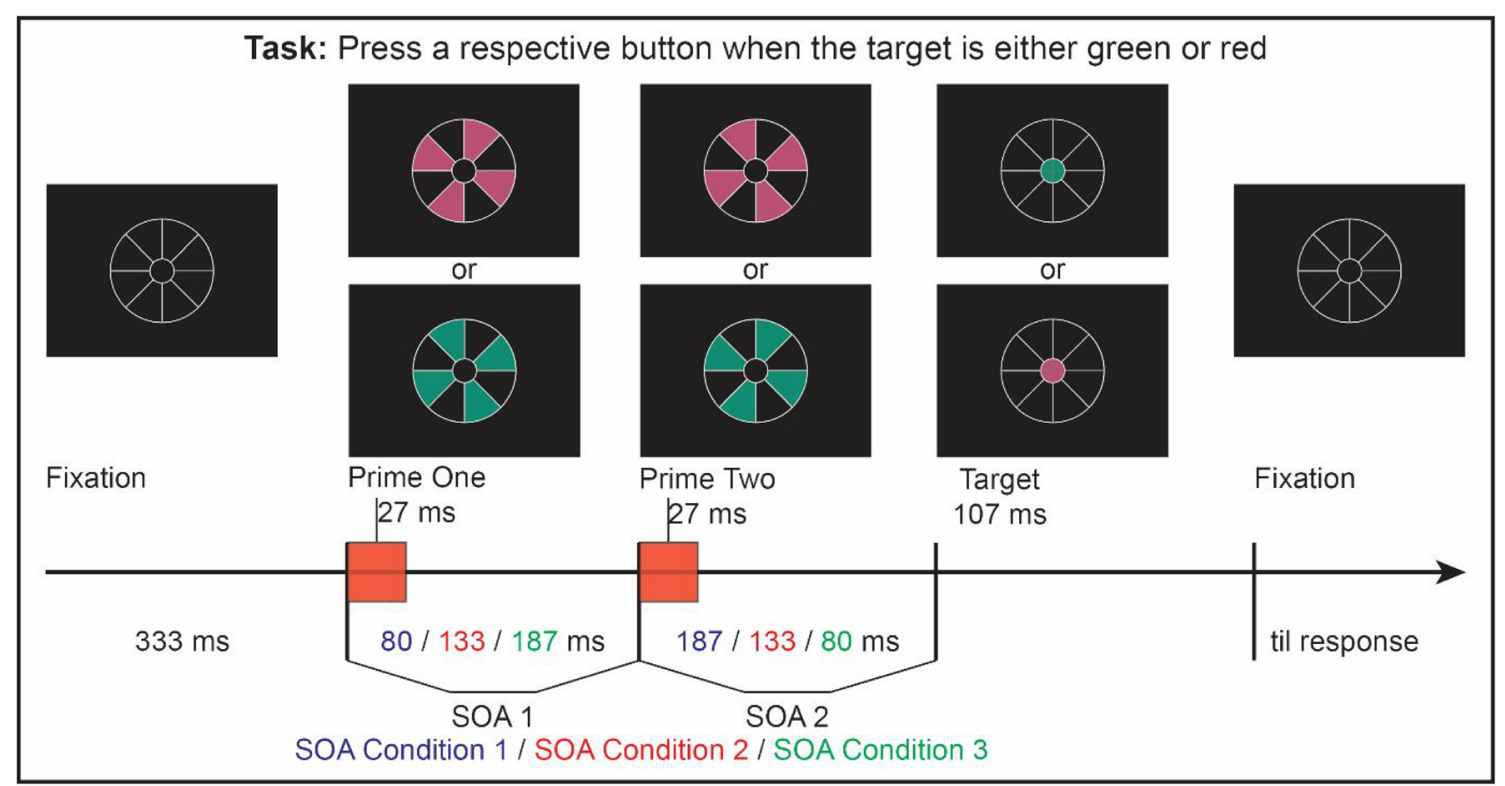

Fig. 6 Stimulus displays and design. After fixating the center of the white lollipop frame a sequence of two primes and a target is presented, with SOA1SOA2 combinations of $80 / 187,133 / 133$, or $187 / 80$ 
Table 3 Selected hazard model for Experiment 2

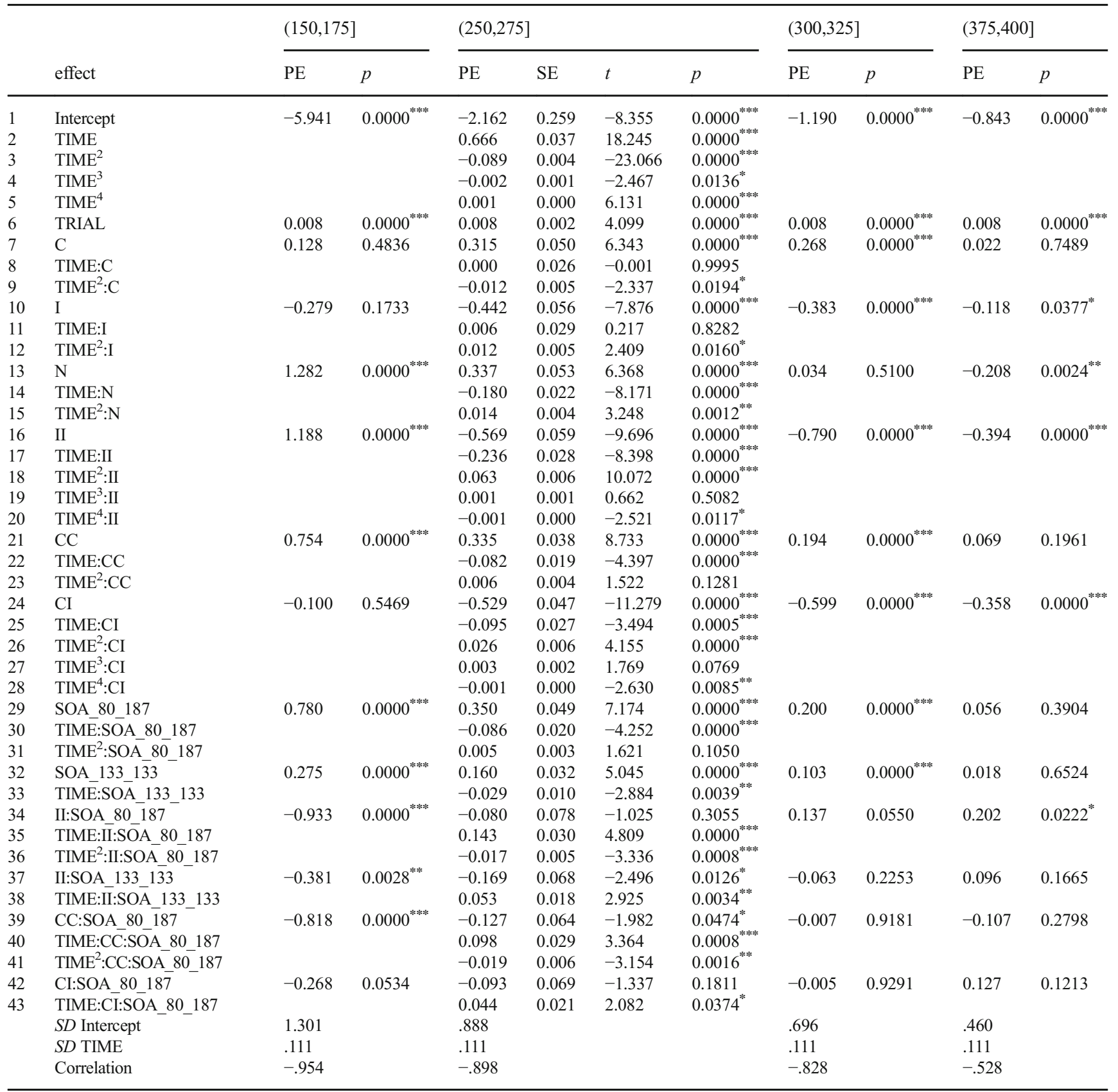

Note. Parameter estimates (PE) and test statistics. During model selection TIME was centered on bin 275. The selected model was refitted three times with TIME centered on bin 175,325 , and 400 , respectively

\section{Experiment 2}

\section{Method}

\section{Participants}

All participants from the first experiment also took part in the second experiment (see Participants section for Experiment 1). Experiment order was counterbalanced.

\section{Apparatus and stimuli}

The same apparatus and stimuli were employed (see Apparatus and Stimuli section in Experiment 1).

\section{Procedure}

Again, each trial began with the onset of the lollipop frame (see Fig. 6). This time, after $333 \mathrm{~ms}$ of fixation, P1 was 
Table 4 Selected $c a(\mathrm{t})$ model for Experiment 2

\begin{tabular}{|c|c|c|c|c|c|c|c|c|c|c|c|}
\hline \multirow{2}{*}{\multicolumn{2}{|c|}{ Effect }} & \multicolumn{2}{|c|}{$(175,200]$} & \multicolumn{4}{|c|}{$(250,275]$} & \multicolumn{2}{|c|}{$(300,325]$} & \multicolumn{2}{|c|}{$(375,400]$} \\
\hline & & PE & $p$ & PE & $\mathrm{SE}$ & $t$ & $p$ & $\mathrm{PE}$ & $p$ & PE & $p$ \\
\hline 1 & Intercept & 1.861 & $0.0000^{* * * *}$ & 2.827 & 0.176 & 16.059 & $0.0000^{* * *}$ & 3.139 & $0.0000^{* * * *}$ & 3.603 & $0.0000^{* * * *}$ \\
\hline 2 & TIME & & & 0.188 & 0.054 & 3.499 & $0.0005^{* * * *}$ & & & & \\
\hline 3 & TIME $^{2}$ & & & -0.025 & 0.013 & -1.968 & $0.0491^{* *}$ & & & & \\
\hline 4 & TIME $^{3}$ & & & 0.005 & 0.003 & 1.877 & 0.0605 & & & & \\
\hline 5 & TIME $^{4}$ & & & 0.000 & 0.000 & -0.784 & 0.4328 & & & & \\
\hline 6 & $\mathrm{C}$ & 0.700 & $0.0378^{*}$ & 0.223 & 0.207 & 1.079 & 0.2807 & -0.095 & 0.5991 & -0.573 & $0.0359^{*}$ \\
\hline 7 & TIME:C & & & -0.159 & 0.061 & -2.599 & $0.0094^{* * *}$ & & & & \\
\hline 8 & I & -3.641 & $0.0000^{* * * *}$ & -1.287 & 0.193 & -6.660 & $0.0000^{* * * *}$ & -0.322 & 0.1310 & 0.218 & 0.4510 \\
\hline 9 & TIME:I & & & 0.603 & 0.095 & 6.345 & $0.0000^{* * * *}$ & & & & \\
\hline 10 & $\mathrm{TIME}^{2}: \mathrm{I}$ & & & -0.060 & 0.020 & -2.998 & $0.0027^{* * *}$ & & & & \\
\hline 11 & $\mathrm{~N}$ & -0.723 & $0.0000^{* * * *}$ & -0.723 & 0.158 & -4.584 & $0.0000^{* * * *}$ & -0.723 & $0.0000^{* * * *}$ & -0.723 & $0.0000^{* * * *}$ \\
\hline 12 & II & -6.031 & $0.0000^{* * * *}$ & -2.997 & 0.173 & -17.332 & $0.0000^{* * * *}$ & -1.590 & $0.0000^{* * * *}$ & -0.402 & 0.0576 \\
\hline 13 & TIME:II & & & 0.827 & 0.064 & 12.927 & $0.0000^{* * * *}$ & & & & \\
\hline 14 & TIME $^{2}$ :II & & & -0.062 & 0.013 & -4.679 & $0.0000^{* * * *}$ & & & & \\
\hline 15 & $\mathrm{CC}$ & 0.961 & $0.0011^{* * *}$ & 0.111 & 0.151 & 0.731 & 0.4645 & 0.229 & 0.1297 & 0.313 & 0.1589 \\
\hline 16 & TIME:CC & & & -0.022 & 0.073 & -0.298 & 0.7660 & & & & \\
\hline 17 & $\mathrm{TIME}^{2}: \mathrm{CC}$ & & & 0.059 & 0.019 & 3.039 & $0.0024^{* *}$ & & & & \\
\hline 18 & $\mathrm{TIME}^{3}: \mathrm{CC}$ & & & -0.009 & 0.004 & -2.637 & $0.0084^{* * *}$ & & & & \\
\hline 19 & CI & -3.660 & $0.0000^{* * * *}$ & -3.678 & 0.188 & -19.545 & $0.0000^{* * * *}$ & -2.233 & $0.0000^{\text {***** }}$ & -1.129 & $0.0000^{* * * *}$ \\
\hline 20 & TIME:CI & & & 0.721 & 0.103 & 7.017 & $0.0000^{* * * *}$ & & & & \\
\hline 21 & $\mathrm{TIME}^{2}: \mathrm{CI}$ & & & 0.072 & 0.025 & 2.823 & $0.0048^{* *}$ & & & & \\
\hline 22 & $\mathrm{TIME}^{3}: \mathrm{CI}$ & & & -0.044 & 0.006 & -6.856 & $0.0000^{* * * *}$ & & & & \\
\hline 23 & $\mathrm{TIME}^{4}: \mathrm{CI}$ & & & 0.004 & 0.001 & 4.837 & $0.0000^{* * * *}$ & & & & \\
\hline 24 & SOA_80_187 & 0.972 & $0.0034^{* *}$ & -0.013 & 0.170 & -0.076 & 0.9396 & -0.386 & $0.0178^{*}$ & -0.574 & $0.0081^{* *}$ \\
\hline 25 & TIME:SOA_80_187 & & & -0.240 & 0.065 & -3.706 & $0.0002^{* * * *}$ & & & & \\
\hline 26 & TIME $^{2}:$ SOA_80_187 & & & 0.028 & 0.022 & 1.291 & 0.1967 & & & & \\
\hline 27 & TIME $^{3}:$ SOA_80_187 & & & 0.000 & 0.004 & -0.128 & 0.8984 & & & & \\
\hline 28 & SOA_133_133 & -0.097 & 0.7321 & 0.051 & 0.162 & 0.317 & 0.7513 & 0.034 & 0.8416 & -0.166 & 0.4399 \\
\hline 29 & TIME:SOA_133_133 & & & 0.015 & 0.056 & 0.260 & 0.7949 & & & & \\
\hline 30 & TIME $^{2}:$ SOA_133_133 & & & -0.012 & 0.012 & -0.978 & 0.3280 & & & & \\
\hline 31 & II:SOA_80_187 & 1.084 & $0.0000^{* * * *}$ & 1.084 & 0.207 & 5.239 & $0.0000^{* * * *}$ & 1.084 & $0.0000^{* * * *}$ & 1.084 & $0.0000^{* * * *}$ \\
\hline 32 & II:SOA_133_133 & 0.724 & $0.0008^{* * * *}$ & 0.724 & 0.215 & 3.365 & $0.0008^{* * * *}$ & 0.724 & $0.0008^{* * * *}$ & 0.724 & $0.0008^{* * * *}$ \\
\hline 33 & CI:SOA_80_187 & -1.783 & $0.0058^{* * *}$ & 1.598 & 0.254 & 6.289 & $0.0000^{* * * *}$ & 1.640 & $0.0000^{* * * *}$ & 0.281 & 0.3841 \\
\hline 34 & TIME:CI:SOA_80_187 & & & 0.369 & 0.119 & 3.089 & $0.0020^{* * *}$ & & & & \\
\hline 35 & TIME $^{2}:$ CI:SOA_80_187 & & & -0.205 & 0.040 & -5.113 & $0.0000^{* * * *}$ & & & & \\
\hline 36 & TIME $^{3}:$ CI:SOA_80_187 & & & 0.016 & 0.006 & 2.538 & $0.0112^{*}$ & & & & \\
\hline 37 & CI:SOA_133_133 & -0.146 & 0.7966 & 0.672 & 0.230 & 2.922 & $0.0035^{* * *}$ & 0.769 & $0.0010^{* *}$ & 0.242 & 0.4493 \\
\hline 38 & TIME:CI:SOA_133_133 & & & 0.138 & 0.111 & 1.242 & 0.2142 & & & & \\
\hline 39 & TIME $^{2}:$ CI:SOA_133_133 & & & -0.045 & 0.023 & -1.987 & $0.0470^{*}$ & & & & \\
\hline & $S D$ Intercept & .231 & & .390 & & & & .496 & & .655 & \\
\hline & $S D$ TIME & .053 & & .053 & & & & .053 & & .053 & \\
\hline & Correlation & .988 & & .996 & & & & .998 & & .999 & \\
\hline
\end{tabular}

Note. Parameter estimates (PE) and test statistics. During model selection TIME was centered on bin 275. The selected model was refitted three times with TIME centered on bin 200,325, and 400, respectively

presented in either red or green for $27 \mathrm{~ms}$, except for the noprime trials, during which all segments remained black. After a P1-P2 SOA of 80,133 , or $187 \mathrm{~ms}$, either a red or green P2 was presented for $27 \mathrm{~ms}$, except for the no-prime and single- 


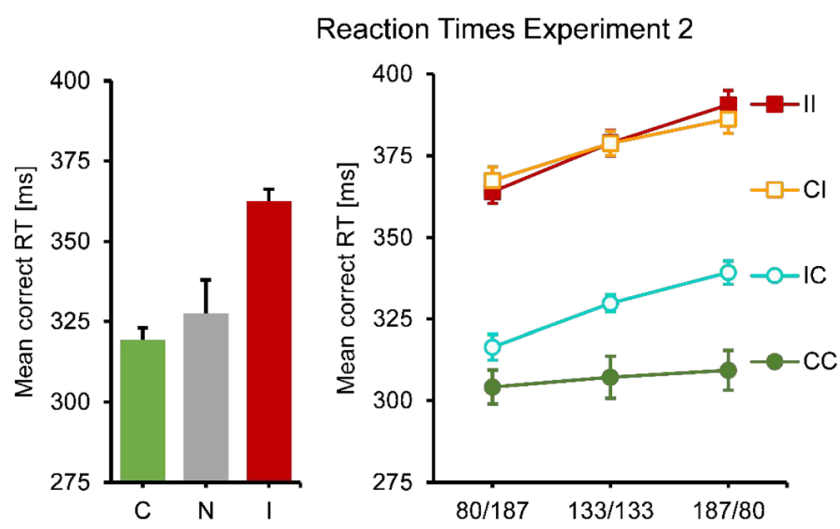

Fig. 7 Mean correct RT results for Experiment 2. No and single-prime conditions: Left panel, error bars resemble the standard error of the mean, consistency conditions on the $x$-axes. Double-prime conditions: Right panel, error bars resemble the standard error of the mean, separate lines for consistency conditions, SOA conditions on the $x$-axes

prime trials, during which all segments remained black. After a P2-T SOA of 187,133 , or $80 \mathrm{~ms}$, respectively, a red or green target was presented. In this experiment, $\mathrm{P} 1-\mathrm{P} 2$ and $\mathrm{P} 2-\mathrm{T}$ SOAs always added up to a P1-T SOA of $267 \mathrm{~ms}$. The target stayed on-screen for $107 \mathrm{~ms}$.

\section{Analysis of mean error rate and mean correct RT}

In a first step, two sets of analyses were performed. First, one-way repeated-measures ANOVAs, with the factor consistency (consistent, inconsistent, no prime), were performed for single-prime and no-prime conditions, one for each of the two dependent variables, RT and ER. A total of 3,600 trials were initially available for analysis. Trials with reaction times faster than $100 \mathrm{~ms}$ or slower than $999 \mathrm{~ms}(0.58 \%)$ were excluded from the analysis. Further, error trials (then $8.16 \%$ ) were excluded from RT analysis.

\section{Error Rates Experiment 2}

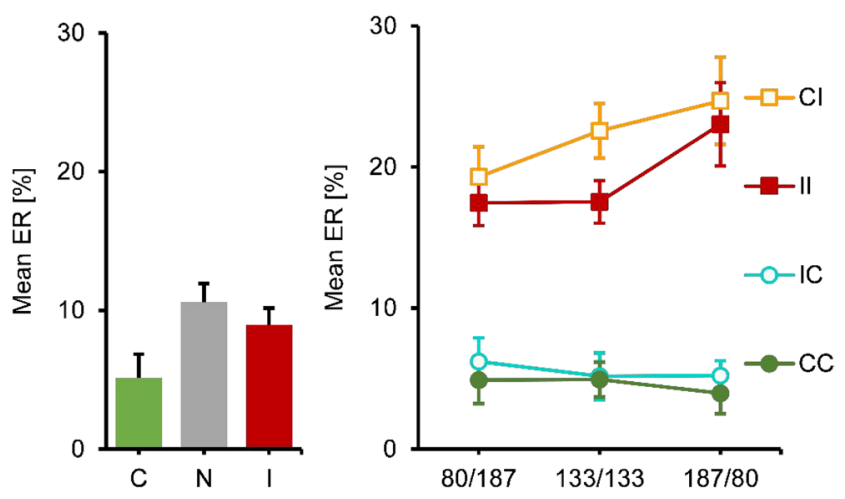

Fig. 8 Mean ER results for Experiment 2. No and single-prime conditions: Left panel, error bars resemble the standard error of the mean, consistency conditions on the $x$-axes. Double-prime conditions: Right panel, error bars resemble the standard error of the mean, separate lines for consistency conditions, SOA conditions on the $x$-axes
Second, two $3(\mathrm{SOA}) \times 4$ (consistency) repeatedmeasures ANOVAs were performed for all doubleprime conditions, one each for RT and ER. Initially, a total of 14,400 trials were available for analysis. Trials with reaction times faster than $100 \mathrm{~ms}$ or slower than $999 \mathrm{~ms}(0.93 \%)$ were excluded from the analysis. Further, error trials (then 12.88\%) were excluded from RT analysis. To follow up on significant interaction effects, one-way repeated-measures ANOVAs, with the four-level factor consistency, were performed separately for each SOA condition.

Greenhouse-Geisser-corrected $p$ values were used. To satisfy ANOVA requirements, error rates were arcsine transformed. Additional within-subjects contrasts were calculated to further investigate significant main effects.

\section{Event history analysis}

First, descriptive statistics were calculated as in Experiment 1 (see Event History Analysis section in Experiment 1). Next, for hazard modeling purposes, we censored the trials at $450 \mathrm{~ms}$ after target onset, and discarded the first five bins, since the most informative events occurred within 125 to $450 \mathrm{~ms}$. The final data set for fitting $h(\mathrm{t})$ models contained 153,286 rows.

Finally, for $c a(t)$ modeling, the original data set was used where each row corresponds to one trial of one participant $(1,500 \times 12=18,000$ trials $)$. Trials with a response latency below $125 \mathrm{~ms}$ or above $450 \mathrm{~ms}$ were deleted (12.36\% of the data), in order to avoid problems of linear separability during model fitting. The final data set for the $c a(\mathrm{t})$ model contained 15,775 rows.

The estimation procedures were the same for both models as in Experiment 1, except that now the IC187/80 condition (P1: inconsistent, P2: consistent, P1P2 SOA: $187 \mathrm{~ms}$, P2-T SOA: $80 \mathrm{~ms}$ ) was chosen as a baseline condition. In summary, with all effects set to zero, the $h(\mathrm{t})$ model's intercept refers to the estimated $\operatorname{cog} \log [h(\mathrm{t})]$, and the $c a(\mathrm{t})$ model's intercept to the estimated $\operatorname{logit}[\mathrm{ca}(\mathrm{t})]$, for bin 275 in Trial 1,000 of the IC$187 / 80$ condition. Again, we refitted the selected model a number of times, with TIME centered each time on another bin (see Tables 3 and 4).

\section{Predictions}

Because P1-T SOAs in Experiment 2 are long, responses are no longer expected to conform to the chase criteria because participants have to wait out the target in order to safeguard against errors provoked by the primes, so that early primes can influence responses only out of the memory buffer that carries information from both primes but is dominated by the second 

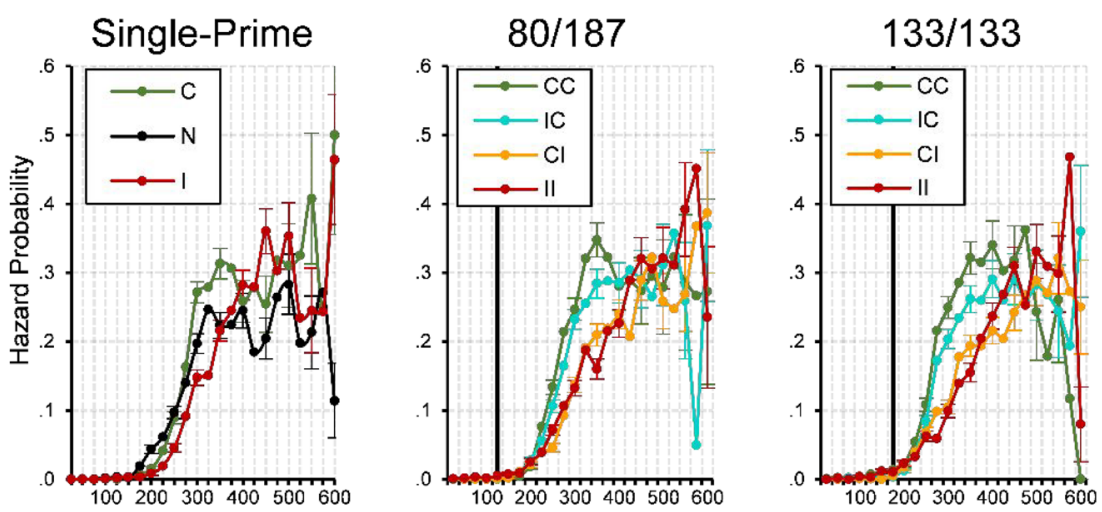

$187 / 80$
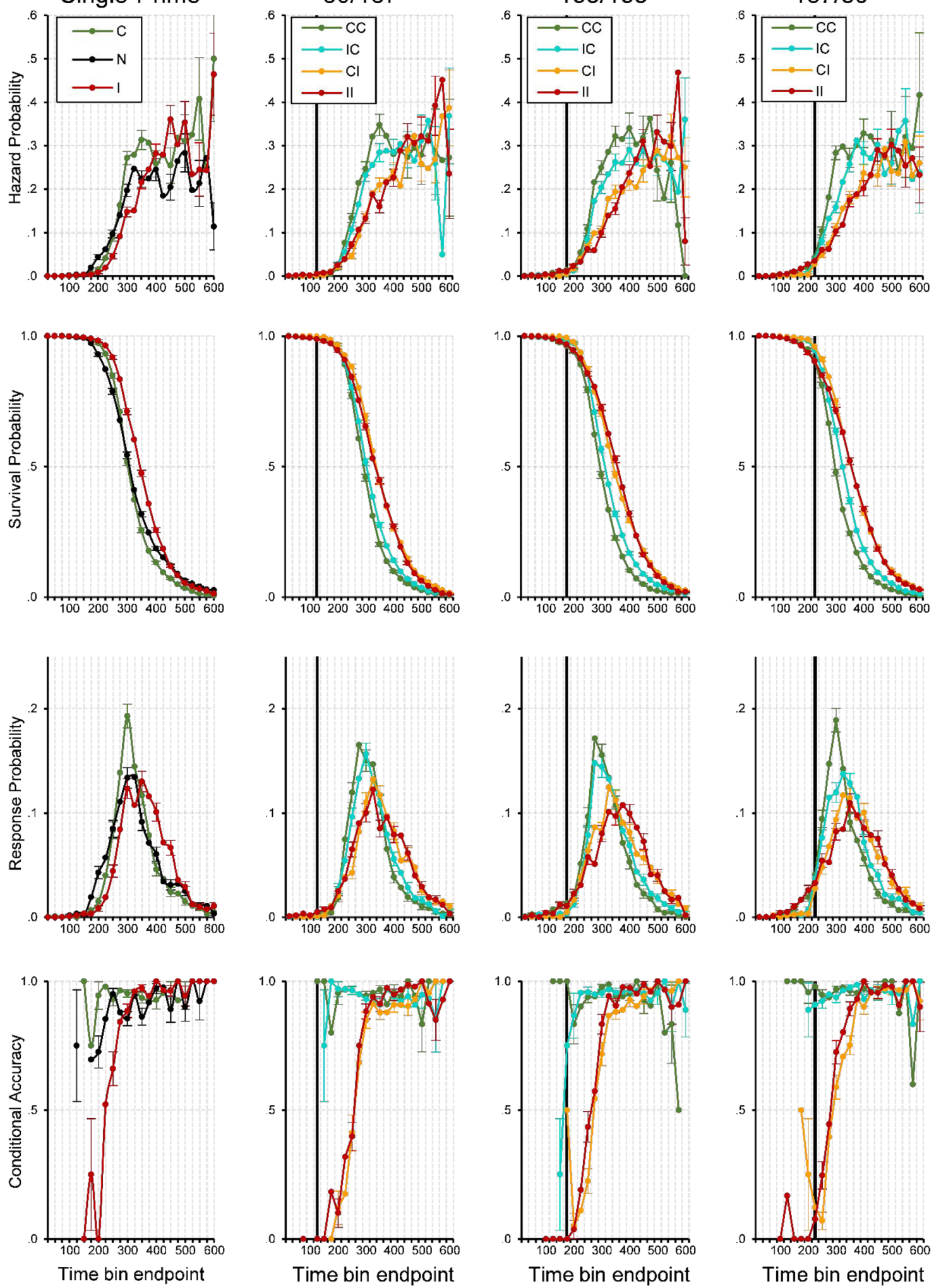

one (Grainger et al., 2013). Therefore, we expected that early responses would no longer be controlled exclusively by the first prime, but jointly by both primes, with the second prime

becoming more dominant as the P2-T SOA increased. The latest responses should be controlled mainly by the target and thus all be correct. 
Fig. 9 Sample-based estimates of $h(\mathrm{t}), S(\mathrm{t}), P(\mathrm{t})$, and $c a(\mathrm{t})$ aggregated across all participants in Experiment 2, for the first 24 bins (or $600 \mathrm{~ms}$ ) after target onset. Bin width equals $25 \mathrm{~ms}$. (First column) Black lines represent the no-prime condition, green lines the consistent single-prime condition, and red lines the inconsistent single-prime condition. (Second to last column) Each column represents a different SOA condition. Green lines represent consistent-consistent conditions, cyan lines inconsistentconsistent conditions, orange lines consistent-inconsistent conditions, red lines inconsistent-inconsistent conditions. Black vertical lines highlight bins at $275-300 \mathrm{~ms}$ after onset of P2. Note that we only plotted a ca(t) estimate if the corresponding hazard for that bin was larger than .003. For better visibility only every second error bar is depicted

\section{Results}

\section{Analysis of mean error rate and mean correct RT}

Analysis of the single prime conditions showed that responses were faster and more accurate when primes were consistent rather than inconsistent. The no-prime condition was intermediate in response times, but higher than the other two in error rate (see Figs. 7 and 8, left panel). One-way repeated-measures ANOVAs showed significant differences in RT, $F(1.34,14.75)=12.60, p=.002$, as well as error rates, $F(1.82,20.02)=3.95, p=.039$. In RTs, the differences between all means were significant, all $p \mathrm{~s} \leq .002$, except the one between consistent and no primes. In ER, only the difference between consistent and no primes was significant, $p=.035$.

In a next step, double-prime conditions were analyzed (see Figs. 7 and 8, right panel). Responses were fastest and most accurate for two consistent primes and slowest and less accurate for two inconsistent primes. The CI condition was virtually identical to the II condition in response times, but slightly higher in error rate. The IC condition was similar to the CC condition in error rates, but slower in terms of response times. In RTs, a two-way repeated-measures ANOVA showed a significant main effect of consistency (with levels CC, CI, IC, II), $F(1.55,17.00)=59.88, p<.001$, a significant main effect of SOA, $F(1.62,17.83)=87.63, p<.001$, and a significant interaction, $F(3.35,36.88)=3.66, p=.018$, that seems to be based on the less steep increase in RT with SOA in the CC condition. We broke down this pattern post hoc into two separate ANOVAs, one for inconsistent and one for consistent second primes. The first one (II versus CI) only showed that response time increased with SOA, $F(1.73,19.01)=87.91, p$ $<.001$. The second test $(\mathrm{CC}$ versus IC) showed that responses were faster for CC than for IC, $F(1.00,11.00)=15.44, p=$ .002 , that RT increased with SOA, $F(1.66,18.30)=15.66, p<$ .001 , and that the increase was steeper for IC than for CC, $F(1.68,18.46)=6.11, p=.012$.

The same strategy was used for the error rates. An ANOVA of all dual-prime conditions showed no main effect of SOA, but a significant main effect of consistency, $F(1.83,20.08)=$ $45.15, p<.001$, and an interaction effect, $F(4.26,46.82)=$
$2.55, p=.048$. The analysis of CC versus IC conditions gave no significant effects, and neither did the analysis of II versus CI conditions.

\section{Event history analysis: Descriptive statistics}

In the single-prime conditions (first column in Fig. 9), the first responses occur after about $200 \mathrm{~ms}$, which is a bit later than in Experiment 1 and in line with the prediction that participants have to safeguard against errors. After that, there is an increase in response hazards that is steeper for consistent than for inconsistent primes, leading to an advantage in mean and median RT. Again, around $400 \mathrm{~ms}$ after target onset, this priming effect is gone. As in Experiment 1, early responses are mostly correct when the single prime is consistent, but incorrect when it is inconsistent, showing that early responses are still determined by the prime, not the target. ${ }^{8}$

Let us now look at the double-prime conditions where the P1-P2 SOA is short and the P2-T SOA is long $(80 / 187$, second column in Fig. 9), so that the impact of the second prime should be high relative to the first prime. Again, although the very earliest responses occur around the same time in all priming conditions, initial response hazards in CI and IC conditions are lower than in $\mathrm{CC}$ and II conditions, reflecting early response competition between both prime-triggered responses (see also the survivor functions). After about $250 \mathrm{~ms}$, both groups begin to differentiate and now follow the order observed in mean RTs: CC, IC, and then CI and II. Again, the most diagnostic information is in the conditional accuracy functions, which show a markedly different pattern than in Experiment 1 . The earliest responses are still predominantly correct when both primes are consistent and predominantly incorrect when both primes are inconsistent, showing that the earliest responses are not determined by the target, but by information in the primes. However, conditional accuracy functions for CC and IC are virtually identical, as are those of II and CI. In other words, the earliest systematic responses reflect only the second prime and not the first, probably because the P1-T SOA is too long. However, if observers would respond faster, we believe that the very first responses would reflect the first prime, just as in Experiment 1.

Although these early effects seem to be driven largely by the second prime, comparison with the $133 / 133$ and $187 / 80$ SOA conditions shows that the effects on hazard cannot be attributed to the second prime alone. While the CC, CI, and II condition show highly similar time courses in every condition, this is not true for the IC condition: The longer the first SOA and the shorter the second one, the more delay appears in condition IC compared with CC (the same effect that is evident in average RT). This effect shows that the first prime has an influence on the timing of the response. Moreover, it 

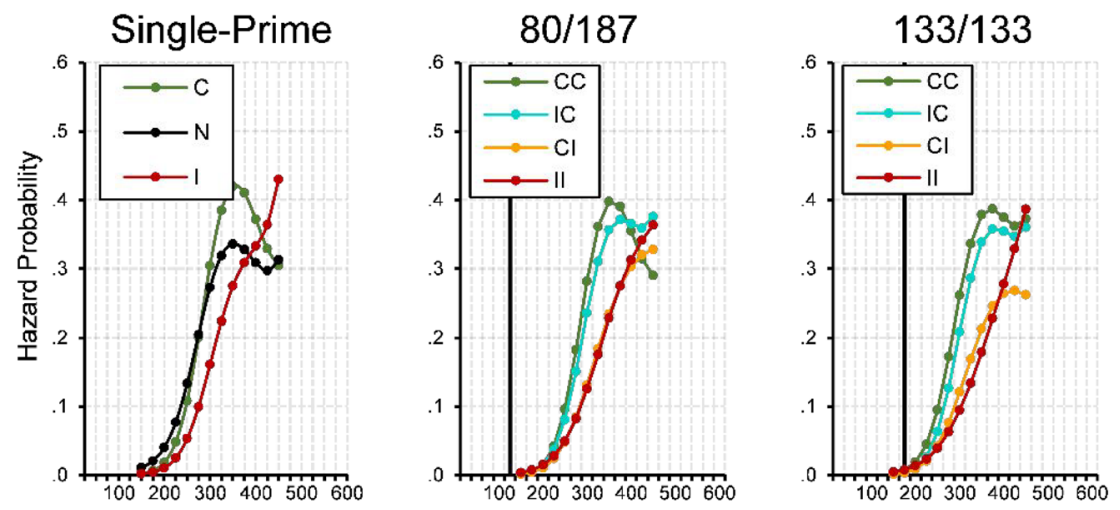

$187 / 80$
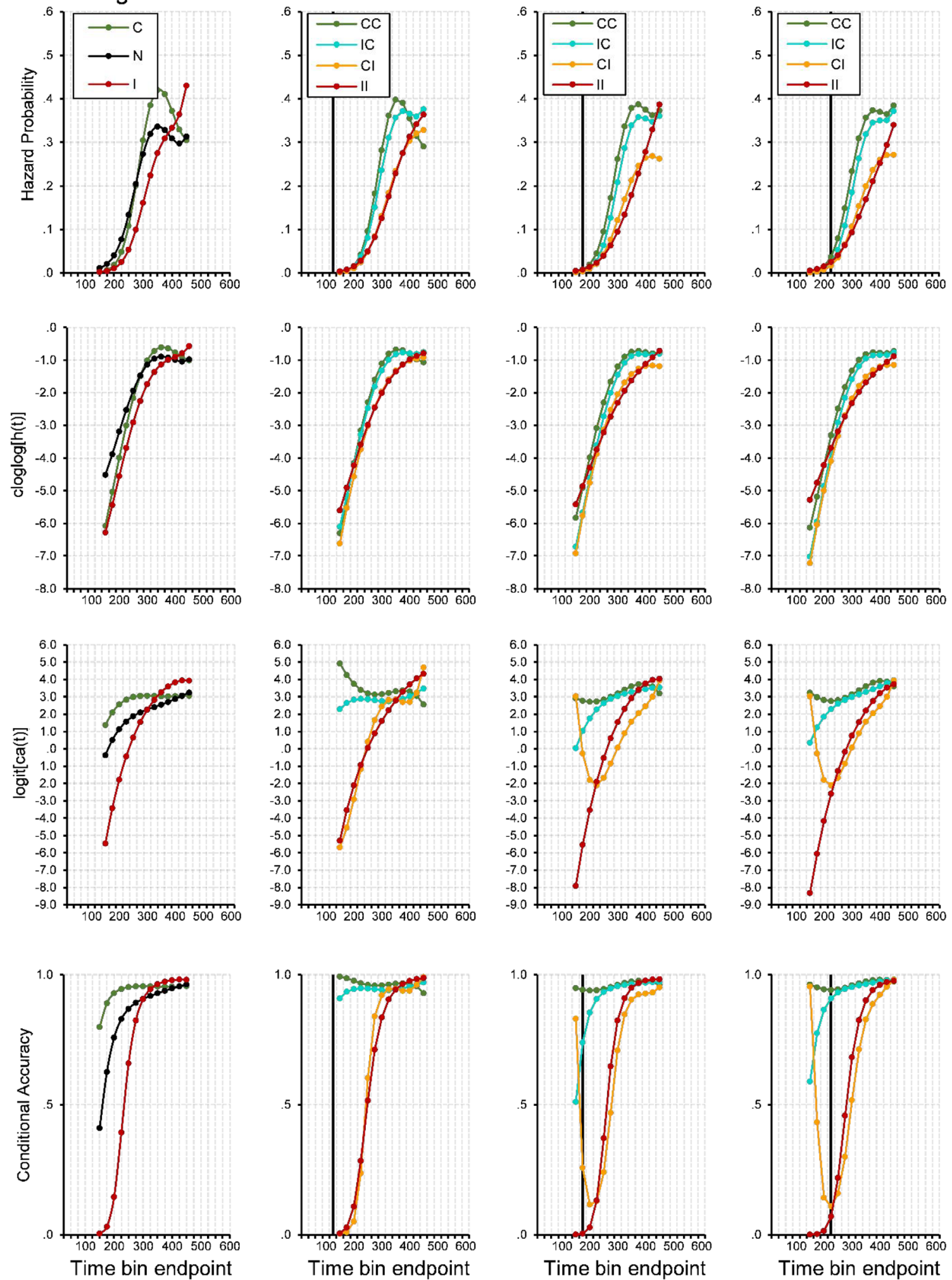

Fig. 10 Model predictions. Predicted hazard (first row), $\operatorname{cog} \log [h(\mathrm{t})]$ (second row), logit $[c a(t)]$ (third row), and conditional accuracy functions (fourth row) for trial 1,000 of Experiment 2. Black vertical lines highlight bins at $275-300 \mathrm{~ms}$ after onset of P2

appears that with shorter P2-T SOA, effects of the second prime become visible in the conditional accuracy functions.
Earliest responses are increasingly more incorrect in the IC condition and increasingly more accurate in the $\mathrm{CI}$ condition. 


\section{Event history analysis: Inferential statistics}

Table 3 shows the selected hazard model, and Table 4 the selected $c a(\mathrm{t})$ model. Figure 10 shows predicted (i.e., modelbased hazard) cloglog[h(t)], logit[ $c a(t)]$ and conditional accuracy functions for Trial 1,000. The first five parameters in Table 3 model the shape of the $\operatorname{cog} \log [h(\mathrm{t})]$ function in the baseline condition, IC-187/80 in Trial 1,000 (see Fig. 10, row 2 , column 4, blue line). Most importantly, compared with condition IC, changing to $\mathrm{CC}$ increases the estimated cloglog-hazard in Bin 275 by .335 units (Parameter 21), changing to CI decreases it by .529 units (Parameter 24), and changing to II decreases it by .569 units (Parameter 16; all $p \mathrm{~s}<.0001)$. While the main effect of CC in Bin 275 decreases over time, the main effects of II and CI increase over time initially (Parameters 17-20, 25-28). Similar to Bin 200 in Experiment 1, in Bin 175 the estimated cloglog-hazard is higher for $\mathrm{CC}$ and II than the mixed conditions IC and CI.

The effect of changing the SOA combination from 187/80 to $133 / 133$ is to increase the estimated cloglog-hazard in the early bins (Parameters 32-33). The estimated cloglog-hazard in these bins increases even further when SOA combination is changed to 80/187 (Parameters 29-31). In other words, response occurrence speeds up with increasing P2-T SOA. The remaining interactions between dual-prime (II, CC, CI), SOA, and TIME (Parameters 34-43) mainly reflect a lower cloglog-hazard in Bin 175, especially for SOA combination 80/187.

The first five parameters in Table 4 model the shape of the $\operatorname{logit}-c a(\mathrm{t})$ function in the baseline condition, IC-187/80 in Trial 1,000 (see Fig. 10, row 3, column 4, blue line). Most importantly, compared with condition $\mathrm{IC}$, changing to $\mathrm{CC}$ increases the estimated logit-ca(t) in Bin 275 by .111 units (Parameter $15 ; p=.4645$ ), changing to $\mathrm{CI}$ decreases it by 3.678 units (Parameter 19; $p<.0001$ ), and changing to II decreases it by 2.997 units (Parameter 12; $p<.0001$ ). These main effects of CC, CI, and II in Bin 275 decrease in magnitude over time (Parameters 12-23).

There is no significant main effect of decreasing the P1-P2 SOA from 187 to 133 (Parameters 28-30), but decreasing it to $80 \mathrm{~ms}$ increases the estimated logit-ca(t) in Bin 200 and decreases the estimated logit-ca $(\mathrm{t})$ for bins $>300 \mathrm{~ms}$ (Parameters 24-27). Finally, there are time-invariant interactions between II and SOA combinations (Parameters 31-32), and timevarying interactions between $\mathrm{CI}$ and SOA combinations (Parameters 33-39), which all increase the estimated logit$c a(\mathrm{t})$ in at least some of the bins.

\section{Summary}

Overall, even with a long P1-T SOA, single-prime conditions produced the common finding in response priming experiments: faster and more accurate responses in consistent trials and slower and less accurate responses in inconsistent trials. Again, when two primes were presented, responses were fastest and most accurate for two consistent primes, and slowest and least accurate for two inconsistent primes. However, under the SOA conditions of Experiment 2, when primes were mixed, we found a clear dominance of the second prime. In particular, CI was almost identical to II, in both response times and error rates. Similarly, IC was almost identical to CC in error rate, but slightly slower. In other words, in both RT and ER the second prime seemed to dominate the response, yet an inconsistent first prime could still slow down response times. This might reflect early response competition due to conflicting prime information in mixed prime conditions.

Again, in order to investigate the temporal dynamics of sequential motor activation, we performed an event history analysis. Altogether, the findings suggest that with prolonged SOAs: (1) The earliest systematic responses were predominantly controlled by the second prime, (2) the slowest responses were controlled by the target, (3) overt responses to the first prime were extremely rare; however, (4) the first prime was able to slow down initial response hazards in mixed prime conditions compared with conditions with identical primes.

\section{General discussion}

The goal of the current study was to investigate (a) whether sequential primes initiate immediate sequential response activation or integrate in a buffer before a response is emitted, (b) whether sequential response activation at short SOAs conforms to the rapid-chase criteria, and (c) how the influence of the first prime changes when the SOAs are prolonged so that participants have to safeguard against early errors from inconsistent primes.

Event history analysis provides substantial evidence that sequential primes initiate strictly sequential response activation at short SOAs (Experiment 1). First, we found that earliest responses were exclusively controlled by the first prime irrespective of the identity or onset time of the second prime, that intermediate responses were influenced by the second prime (with the magnitude and timing of this effect depending on the second prime's onset time), and that only late responses were controlled by the actual target. This strongly supports the notion of feedforward and sequential activation, and is in line with previous findings that first responses are exclusively triggered by prime properties, independent of the target, and only later responses are influenced by target properties (Eimer \& Schlaghecken, 1998; Grainger et al., 2013; Schmidt \& Schmidt, 2010; Schmidt, 2002; Schmidt \& Schmidt, 2009; Vath \& Schmidt, 2007). Thus, the data adhere to the chase criteria proposed by T. Schmidt (2014): (1) The first prime 
rather than the target or subsequent prime signals determine the onset and initial direction of the response; (2) target and second prime influence the response before it is completed; (3) movement kinematics initially depend on characteristics of the first prime only and are independent of all characteristics of target and subsequent prime signals.

Second, as mentioned before (see Multiple-Prime Paradigm section), a simple feedforward-sweep model seems to account very well for response priming effects at short SOAs (up to $100 \mathrm{~ms}$ ). However, priming effects at longer SOAs are more plausibly carried by the content of a response buffer that carries information from both primes but is dominated by the second one (Grainger et al., 2013). This notion is supported by our findings. When SOAs were long (Experiment 2), we found that early systematic responses were predominantly triggered by the second prime's identity and that later responses were triggered by the target's identity. In contrast to the first experiment, we found that overt responses to the first prime were extremely rare, but we identified an indirect, covert influence of the first prime's identity on motor response activation, as there were signs of response competition due to conflicting prime information in mixed prime conditions. This strongly suggests that information of a first prime was indeed maintained in a memory buffer and could influence the response that is otherwise dominated by the second prime. Future computational models of decisionmaking (cf. Mattler \& Palmer, 2012; Schmidt \& Schmidt, 2018; Schubert, Palazova, \& Hutt, 2013; Ulrich, Schröter, Leuthold, \& Birngruber, 2015; Vorberg et al., 2003) should test whether the observed hazard and conditional accuracy functions can be simulated with or without a memory buffer.

It is important to point to the different insights that can be gained from an ANOVA on mean correct RT, versus an event history analysis. First, in accordance with the conclusions from previous findings (Breitmeyer \& Hanif, 2008; Grainger et al., 2013), a second prime dominates the priming effect in mean correct RTs and ER, at least for short interprime intervals. However, the event history analysis showed that the first prime dominated the motor response in the earliest bins (Experiment 1). Thus, in contrast to Breitmeyer and Hanif (2008), this suggests that the second prime does not update and override the effects of the first prime, but that both prime-triggered motor responses are competing in mixed conditions, and under the right SOA setup, even the first prime is able to dominate the motor response.

Second, when SOAs were long (Experiment 2), we found an even clearer dominance of the second prime since participants seemed to safeguard against early errors provoked by the first prime by waiting out the target. Although response accuracy was entirely dominated by the second prime, RT analysis revealed that an inconsistent first prime in IC conditions could still slow down responses compared with consistent-only conditions. The event history analysis confirmed that early hazards were lower in mixed prime conditions compared with identical prime conditions. We propose that this is due to response competition created by conflicting prime information. Further, this effect increased with prolonged SOAs between primes, again reflecting a reduced dominance of the second prime due to an increase of the first prime's effect. Thus, the first prime can still influence the motor response with long SOAs. Note that these systematic differences between SOA ranges imply that long and short SOAs should not be mixed within the same experiment, since the presence of long SOAs would enforce a strategy of waiting out the target even in trials where the SOA is short (Schmidt, Haberkamp, \& Schmidt, 2011).

When we compare SOA combination $187 / 80$ of Experiment 2 with SOA combination 27/80 of Experiment 1 , we see that $\mathrm{P} 2$ dominated behavior more in the former than in the latter condition. Therefore, in line with Grainger et al. (2013), we propose that the first prime can influence the response only out of a memory buffer in Experiment 2, since prime information seemed to be kept active for a prolonged period of time without activating a response on its own.

Importantly, we designed our lollipop stimulus in such a way as to minimize masking effects (no spatial overlap) and Simon/ flanker effects (prime information is presented at both sides of the target). However, it is unclear if active response inhibition was playing a role in the generation of the behavior in Experiment 2. Panis and Schmidt (2016) and Schmidt, Hauch, and Schmidt (2015) showed that a second stimulus can trigger active and selective inhibition of the response triggered by a first stimulus, within about $360 \mathrm{~ms}$. For example, for SOA combinations 133/ 133 and $187 / 80$ we see that CI has a lower conditional accuracy than II for bins after $225 \mathrm{~ms}$. This might be caused by active inhibition of the first compatible response, creating an even stronger activation of the incompatible response channel in condition CI than in II. Future modeling studies should investigate this issue further.

More generally, the information obtained from an event history analysis can provide strong constraints for computational models of the underlying sensory integration, decision, and cognitive control processes (Panis, Moran, Wolkersdorfer, \& Schmidt, 2020). For example, existing models differ in (a) whether sensory integration is perfect (e.g., the drift-diffusion model; Ratcliff \& Rouder, 1998) or leaky (e.g., the leaky competing accumulator model of Usher \& McClelland, 2001), (b) whether the response criterion is fixed (e.g., Poisson accumulator models; Schmidt \& Schmidt, 2018; Schubert et al., 2013; Vorberg et al., 2003) or variable (the urgency gating model of Cisek, Puskas, \& El-Murr, 2009), and (c) whether classic computational principles (e.g., the Bayesian reader model of Norris, 2006) or dynamic principles (e.g., the dynamic field theory of Schöner, Spencer, \& The DFT Research Group, 2016) are used (e.g., see Carland, Thura, \& Cisek, 2019, for a discussion of these issues). Comparing empirical and simulated data from such models using event history analysis will allow 
future studies to better select between and validate the different computational models available in the literature.

While behavioral experiments are informative, they allow only indirect inferences about the underlying neural correlates. Ultimately, one wants to complement the behavioral data with physiological data such as EEG, fMRI, single-cell data, and so forth. Note that hazard modeling allows incorporating timevarying explanatory covariates such as heart rate, EEG signal amplitude, and gaze location (Allison, 2010), which is useful for cognitive psychophysiology (Meyer, Osman, Irwin, \& Yantis, 1988).

In summary, the current study provides substantial evidence that sequential primes actually initiate sequential response activation, and that this sequence conforms to the chase criteria at short SOAs. However, when SOAs are prolonged participants have to delay their responses, the first prime seems to influence responses out of a memory buffer.

Open practices statement All data, materials and analyses are available from the authors upon request. All independent and dependent variables are identified. Preregistration was not employed.

Acknowledgements Part of this work was supported by the Deutsche Forschungsgemeinschaft (DFG, German Research Foundation)Projektnummer PA 2947/1-1 (to S.P.).

Funding Information Open Access funding provided by Projekt DEAL.

\section{Compliance with ethical standards}

\section{Declarations of interest None.}

Author contributions We thank Stefan Hellrigel, Larissa Leist, Stefanie Scülfort, and Thomas Wegner for their contributions in designing the paradigm.

Open Access This article is licensed under a Creative Commons Attribution 4.0 International License, which permits use, sharing, adaptation, distribution and reproduction in any medium or format, as long as you give appropriate credit to the original author(s) and the source, provide a link to the Creative Commons licence, and indicate if changes were made. The images or other third party material in this article are included in the article's Creative Commons licence, unless indicated otherwise in a credit line to the material. If material is not included in the article's Creative Commons licence and your intended use is not permitted by statutory regulation or exceeds the permitted use, you will need to obtain permission directly from the copyright holder. To view a copy of this licence, visit http://creativecommons.org/licenses/by/4.0/.

\section{References}

Allison, P. D. (1982). Discrete-time methods for the analysis of event histories. Sociological Methodology, 13, 61-98. https://doi.org/10. 2307/270718

Allison, P. D. (2010). Survival analysis using SAS: A practical guide (2nd ed.). Cary, NC: SAS Institute Inc.
Barr, D. J., Levy, R., Scheepers, C., \& Tily, H. J. (2013). Random effects structure for confirmatory hypothesis testing: Keep it maximal. Journal of Memory and Language, 68(3), 255-278. https://doi. org/10.1016/j.jml.2012.11.001

Brainard, D. H. (1997). The Psychophysics Toolbox. Spatial Vision, 10, 433-436.

Breitmeyer, B. G., \& Hanif, W. (2008). "Change of mind" within and between nonconscious (masked) and conscious (unmasked) visual processing. Consciousness and Cognition, 17, 254-266. https://doi. org/10.1016/j.concog.2007.08.001

Bullier, J. (2001). Integrated model of visual processing. Brain Research Reviews, 36(2/3), 96-107. https://doi.org/10.1016/S0165-0173(01) 00085-6

Burle, B., Vidal, F., Tandonnet, C., \& Hasbroucq, T. (2004). Physiological evidence for response inhibition in choice reaction time tasks. Brain and Cognition, 56(2), 153-164. https://doi.org/ 10.1016/j.bandc.2004.06.004

Carland, M. A., Thura, D., \& Cisek, P. (2019). The urge to decide and act: Implications for brain function and dysfunction. The Neuroscientist, 25(5), 491-511. https://doi.org/10.1177/1073858419841553

Cisek, P., Puskas, G. A., \& El-Murr, S. (2009). Decisions in changing conditions: The urgency-gating model. Journal of Neuroscience, 29(37), 11560-11571. https://doi.org/10.1523/JNEUROSCI.184409.2009

Cunnings, I. (2012). An overview of mixed-effects statistical models for second language researchers. Second Language Research, 28(3), 369-382. https://doi.org/10.1177/2F0267658312443651

Eimer, M., \& Schlaghecken, F. (1998). Effects of masked stimuli on motor activation: Behavioral and electrophysiological evidence. Journal of Experimental Psychology: Human Perception and Performance, 24(6), 1737-1747. https://doi.org/10.1037/00961523.24.6.1737

Eisenhart, C. (1962). Realistic evaluation of the precision and accuracy of instrument calibration systems. In H. H. Ku (Ed.), Precision measurement and calibration (pp. 21-48). Washington, DC: National Bureau of Standards.

Fernández-López, M., Marcet, A., \& Perea, M. (2019). Can response congruency effects be obtained in masked priming lexical decision? Journal of Experimental Psychology: Learning, Memory, and Cognition, 45(9), 1683-1702. https://doi.org/10.1037/xlm0000666

Ferrand, L., \& Grainger, J. (1992). Phonology and orthography in visual word recognition: Evidence from masked non-word priming. The Quarterly Journal of Experimental Psychology Section A, 45(3), 353-372. https://doi.org/10.1080/2F02724989208250619

Grainger, J. E., Scharnowski, F., Schmidt, T., \& Herzog, M. H. (2013). Two primes priming: Does feature integration occur before response activation? Journal of Vision, 13(8), 19 .https://doi.org/10.1167/13. 8.19

Jaśkowski, P., Skalska, B., \& Verleger, R. (2003). How the self controls its "automatic pilot" when processing subliminal information. Journal of Cognitive Neuroscience, 15(6), 911-920. https://doi. org/10.1162/089892903322370825

Kleiner, M., Brainard, D., Pelli, D., Ingling, A., Murray, R., \& Broussard, C. (2007). What's new in Psychtoolbox-3? Perception. 36(14), 1-16.

Klotz, W., \& Neumann, O. (1999). Motor activation without conscious discrimination in metacontrast masking. Journal of Experimental Psychology: Human Perception and Performance, 25(4), 976992. https://doi.org/10.1037/0096-1523.25.4.976

Klotz, W., \& Wolff, P. (1995). The effect of a masked stimulus on the response to the masking stimulus. Psychological Research, 58(2), 92-101. https://doi.org/10.1007/BF00571098

Lamme, V. A.F., \& Roelfsema, P. R. (2000). The distinct modes of vision offered by feedforward and recurrent processing. Trends in Neurosciences, 23(11), 571-579. https://doi.org/10.1016/S01662236(00)01657-X 
Li, X., Liang, Z., Kleiner, M., \& Lu, Z.-L. (2010). RTbox: A device for highly accurate response time measurements. Behavior Research Methods, 42, 212-225. https://doi.org/10.3758/BRM.42.1.212

Luce, R. D. (1986). Response times: Their role in inferring elementary mental organization. New York, NY: Oxford University Press.

Mattler, U., \& Palmer, S. (2012). Time course of free-choice priming effects explained by a simple accumulator model. Cognition, 123(3), 347-360. https://doi.org/10.1016/j.cognition.2012.03.002

Matuschek, H., Kliegl, R., Vasishth, S., Baayen, H., \& Bates, D. (2017). Balancing Type I error and power in linear mixed models. Journal of Memory and Language, 94, 305-315. https://doi.org/10.1016/j.jml. 2017.01.001

Meyer, D. E., Osman, A. M., Irwin, D. E., \& Yantis, S. (1988). Modern mental chronometry. Biological psychology, 26(1/3), 3-67. doi: https://doi.org/10.1016/0301-0511(88)90013-0

Miller, J. (1982). Divided attention: Evidence for coactivation with redundant signals. Cognitive Psychology, 14(2), 247-279. https://doi. org/10.1016/0010-0285(82)90010-X

Norris, D. (2006). The Bayesian reader: Explaining word recognition as an optimal Bayesian decision process. Psychological Review, 113(2), 327. Retrieved from https://doi.org/10.1037/0033-295X.113.2.327

Panis, S., \& Hermens, F. (2014). Time course of spatial contextual interference: Event history analyses of simultaneous masking by nonoverlapping patterns. Journal of Experimental Psychology: Human Perception and Performance, 40(1), 129-144. https://doi.org/10.1037/a0032949

Panis, S., Moran, R., Wolkersdorfer, M. P., \& Schmidt, T. (2020). Studying the dynamics of visual search behavior using RT hazard and micro-level speed-accuracy tradeoff functions: A role for recurrent object recognition and cognitive control processes. Attention, Perception, \& Psychophysics, 1-26. Advance online publication. https://doi.org/10.3758/s13414-019-01897-z

Panis, S., \& Schmidt, T. (2016). What is shaping RT and accuracy distributions? Active and selective response inhibition causes the negative compatibility effect. Journal of Cognitive Neuroscience, 28(11), 1651-1671. https://doi.org/10.1162/jocn_a_00998

Panis, S., Torfs, K., Gillebert, C. R., Wagemans, J., \& Humphreys, G. W. (2017). Neuropsychological evidence for the temporal dynamics of category-specific naming. Visual Cognition, 25(1/3), 79-99. https:// doi.org/10.1080/13506285.2017.1330790

Panis, S., \& Wagemans, J. (2009). Time-course contingencies in perceptual organization and identification of fragmented object outlines. Journal of Experimental Psychology: Human Perception and Performance, 35(3), 661-687. https://doi.org/10.1037/a0013547

Pelli, D. G. (1997). The VideoToolbox software for visual psychophysics: Transforming numbers into movies. Spatial Vision, 10, 437-442.

Praamstra, P., \& Seiss, E. (2005). The neurophysiology of response competition: Motor cortex activation and inhibition following subliminal response priming. Journal of Cognitive Neuroscience, 17(3), 483493. https://doi.org/10.1162/0898929053279513

R Core Team (2014). R: A language and environment for statistical computing. R Foundation for Statistical Computing, Vienna, Austria. URL http://www.R-project.org/

Ratcliff, R., \& Rouder, J. N. (1998). Modeling response times for twochoice decisions. Psychological Science, 9, 347-356. https://doi. org/10.1111/1467-9280.00067

Rosenbaum, D. A. (1983). The movement precuing technique: Assumptions, applications, and extensions. Advances in Psychology, 12, 231-274. https://doi.org/10.1016/S0166-4115(08)61994-9

Schacter, D. L., \& Buckner, R. L. (1998). Priming and the brain. Neuron, 20(2), 185-195. https://doi.org/10.1126/science.281.5380.1188

Schmidt, F., Haberkamp, A., \& Schmidt, T. (2011). Dos and don'ts in response priming research. Advances in Cognitive Psychology, 7, 120-131. https://doi.org/10.2478/v10053-008-0092-2

Schmidt, F., \& Schmidt, T. (2010). Feature-based attention to unconscious shapes and colors. Attention, Perception, \& Psychophysics, 72(6), 1480-1494. https://doi.org/10.3758/APP.72.6.1480
Schmidt, T. (2002). The finger in flight: Real-time motor control by visually masked color stimuli. Psychological Science, 13(2), 112118. https://doi.org/10.1111/2F1467-9280.00421

Schmidt, T. (2014). Behavioral criteria of feedforward processing in rapid-chase theory: Some formal considerations. Retrieved from https://arxiv.org/abs/1405.5795

Schmidt, T., Haberkamp, A., Veltkamp, G. M., Weber, A., SeydellGreenwald, A., \& Schmidt, F. (2011). Visual processing in rapidchase systems: Image processing, attention, and awareness. Frontiers in Psychology, 2, 169. https://doi.org/10.3389/fpsyg.2011.00169

Schmidt, T., Hauch, V., \& Schmidt, F. (2015). Mask-triggered thrust reversal in the negative compatibility effect. Attention, Perception, \& Psychophysics, 77(7), 2377-2398. https://doi.org/10.3758/s13414-015-0923-4

Schmidt, T., Niehaus, S., \& Nagel, A. (2006). Primes and targets in rapid chases: Tracing sequential waves of motor activation. Behavioral Neuroscience, 120(5), 1005-1016. https://doi.org/10.1037/07357044.120.5.1005

Schmidt, T., \& Schmidt, F. (2009). Processing of natural images is feedforward: A simple behavioral test. Attention, Perception, \& Psychophysics, 71(3), 594-606. https://doi.org/10.3758/APP.71.3.594

Schmidt, T., \& Schmidt, F. (2018). An accumulator model for primes and targets with independent response activation rates: Basic equations for average response times. Retrieved from https://arxiv.org/ftp/ arxiv/papers/1804/1804.08513.pdf

Schöner, G., Spencer, J., \& The DFT Research Group. (2016). Dynamic thinking: A primer on dynamic field theory. New York, NY: Oxford University Press.

Schubert, T., Palazova, M., \& Hutt, A. (2013). The time course of temporal attention effects on nonconscious prime processing. Attention, Perception, \& Psychophysics, 75(8), 1667-1686. https://doi.org/10. 3758/s13414-013-0515-0

Singer, J. D., \& Willett, J. B. (2003). Applied longitudinal data analysis: Modeling change and event occurrence. New York, NY: Oxford University Press.

Smith, P. L., \& Little, D. R. (2018). Small is beautiful: In defense of small-N designs. Psychonomic Bulletin and Review, 25, 20832101. https://doi.org/10.3758/s13423-018-1451-8

Ulrich, R., Schröter, H., Leuthold, H., \& Birngruber, T. (2015). Automatic and controlled stimulus processing in conflict tasks: Superimposed diffusion processes and delta functions. Cognitive Psychology, 78, 148174. https://doi.org/10.1016/j.cogpsych.2015.02.005

Usher, M., \& McClelland, J. L. (2001). The time course of perceptual choice: The leaky, competing accumulator model. Psychological Review, 108(3), 550. https://doi.org/10.1037/0033-295X.108.3.550

VanRullen, R., \& Koch, C. (2003). Visual selective behavior can be triggered by a feed-forward process. Journal of Cognitive Neuroscience, 15(2), 209-217. https://doi.org/10.1162/089892903321208141

Vath, N., \& Schmidt, T. (2007). Tracing sequential waves of rapid visuomotor activation in lateralized readiness potentials. Neuroscience, 145(1), 197208. https://doi.org/10.1016/j.neuroscience.2006.11.044

Vorberg, D., Mattler, U., Heinecke, A., Schmidt, T., \& Schwarzbach, J. (2003). Different time courses for visual perception and action priming. Proceedings of the National Academy of Sciences of the United States of America, 100(10), 6275-6280. https://doi.org/10.1073/ pnas. 0931489100

Wiggs, C. L., \& Martin, A. (1998). Properties and mechanisms of perceptual priming. Current Opinion in Neurobiology, 8(2), 227-233. https://doi.org/10.1016/S0959-4388(98)80144-X

Zuur, A. F., \& Ieno, E. N. (2016). A protocol for conducting and presenting results of regression-type analyses. Methods in Ecology and Evolution, 7(6), 636-645. https://doi.org/10.1111/2041-210X.12577

Publisher's note Springer Nature remains neutral with regard to jurisdictional claims in published maps and institutional affiliations. 